\title{
Tau is not necessary for amyloid- $\beta$-induced synaptic and memory impairments
}

\author{
Daniela Puzzo, ${ }^{1,2}$ Elentina K. Argyrousi, ${ }^{3,4}$ Agnieszka Staniszewski, ${ }^{3,4}$ Hong Zhang, ${ }^{3,4}$ Elisa Calcagno, ${ }^{3,4}$ Elisa Zuccarello, ${ }^{3,4}$ \\ Erica Acquarone, ${ }^{3,4}$ Mauro Fa', 3,4 Domenica D. Li Puma, ${ }^{5,6}$ Claudio Grassi,, ${ }^{5,6}$ Luciano D’Adamio,, Nicholas M. Kanaan, ${ }^{8}$ \\ Paul E. Fraser, ${ }^{9,10}$ and Ottavio Arancio ${ }^{3,4}$
}

1Department of Biomedical and Biotechnological Sciences, University of Catania, Catania, Italy. ${ }^{2}$ Oasi Research Institute-IRCCS, Troina, Italy. ${ }^{3}$ Department of Pathology and Cell Biology, Taub Institute for Research on Alzheimer's Disease and the Aging Brain, and ${ }^{4}$ Department of Medicine, Columbia University, New York, New York, USA. ${ }^{5}$ Department of Neuroscience, Università Cattolica del Sacro Cuore, Rome, Italy. ${ }^{6}$ Fondazione Policlinico A. Cemelli-IRCCS, Rome, Italy. ${ }^{7}$ Department of Pharmacology, Physiology and Neuroscience, Rutgers University, Newark, New Jersey, USA. ${ }^{8}$ Department of Translational Neuroscience, College of Human Medicine, Michigan State University, Grand Rapids, Michigan, USA. ${ }^{9}$ Tanz Centre for Research in Neurodegenerative Diseases, and ${ }^{10}$ Department of Medical Biophysics, University of Toronto, Toronto, Canada.

\begin{abstract}
The amyloid hypothesis posits that the amyloid-beta $(A \beta)$ protein precedes and requires microtubule-associated protein tau in a sort of trigger-bullet mechanism leading to Alzheimer's disease (AD) pathology. This sequence of events has become dogmatic in the $A D$ field and is used to explain clinical trial failures due to a late start of the intervention when $A \beta$ already activated tau. Here, using a multidisciplinary approach combining molecular biological, biochemical, histopathological, electrophysiological, and behavioral methods, we demonstrated that tau suppression did not protect against $A \beta$-induced damage of long-term synaptic plasticity and memory, or from amyloid deposition. Tau suppression could even unravel a defect in basal synaptic transmission in a mouse model of amyloid deposition. Similarly, tau suppression did not protect against exogenous oligomeric tau-induced impairment of long-term synaptic plasticity and memory. The protective effect of tau suppression was, in turn, confined to short-term plasticity and memory. Taken together, our data suggest that therapies downstream of $A \beta$ and tau together are more suitable to combat $A D$ than therapies against one or the other alone.
\end{abstract}

\section{Introduction}

The amyloid cascade hypothesis dominates in the Alzheimer's disease $(\mathrm{AD})$ field. It posits that amyloid- $\beta(\mathrm{A} \beta)$ and tau proteins are placed in a series with $A \beta$ upstream of tau, in a sort of trigger-bullet mechanism. In support of this hypothesis, a decrease or genetic suppression of tau prevents $A \beta$-induced synaptic damage, neuronal toxicity, and axonal defects (1-3). Moreover, reducing tau levels counteracts $A \beta$-induced synaptic plasticity and behavioral abnormalities $(1,4,5)$ and spreading of the pathology throughout the brain (6). Consistent with these findings, the expression of human WT but not mutant $\mathrm{N} 296 \mathrm{H}$ tau rescues the A $\beta$-induced inhibition of long-term potentiation (LTP) in tau knockout (KO) mice (7). As a consequence of the amyloid cascade hypothesis, many scientists have ascribed the failure of anti-A $\beta$ clinical trials to a late intervention in the disease development when $A \beta$ has already triggered tau, producing pathology independently of $A \beta$. Indeed, a large number of studies are currently focusing either on starting anti-A $\beta$ therapies early in the disease progression when tau has not yet been triggered by $\mathrm{A} \beta$, or on the role of tau in $\mathrm{AD}$ pathogenesis, with the ultimate goal of arresting the disease by acting onto tau.

Conflict of interest: The authors have declared that no conflict of interest exists Copyright: (5) 2020, American Society for Clinical Investigation.

Submitted: February 4, 2020; Accepted: June 10, 2020; Published: August 10, 2020.

Reference information: J Clin Invest. 2020;130(9):4831-4844.

https://doi.org/10.1172/JCl137040.
Numerous studies suggest that $A \beta$ and tau have a common toxicity mechanism. Both proteins (a) are released upon neuronal activity (8-13), (b) permeate neuronal and glial cells (14-20), (c) undergo spreading throughout the brain (21), (d) impair synaptic function and memory $(10,22,23)$, and (e) need cellular prion protein for disrupting hippocampal synaptic plasticity (24). Most importantly, $A \beta$ and tau cooperate to produce behavioral deficits, synaptic dysfunction, and downregulation of transcription of genes involved in synaptic function $(10,25)$. Interestingly, concurrent administration of low subtoxic doses of oligomers of $A \beta$ $(\mathrm{oA} \beta)$ and tau (oTau) produces an immediate disruption of memory and hippocampal LTP, a type of synaptic plasticity thought to underlie memory formation (10), supporting the idea that the 2 proteins might act in parallel to exert their detrimental effects (26). Consistent with this idea, amyloid precursor protein (APP) is necessary for the detrimental effect of $A \beta$ and tau onto LTP and memory (19) with both $A \beta$ and tau binding APP $(19,27-31)$. Taken together, these results beg the question of whether $A \beta$ and tau are placed in a series or in parallel in the processes leading to synaptic dysfunction and memory loss. Addressing this question is of high relevance in the field because if the 2 proteins act in parallel, both anti-A $\beta$ and anti-tau therapies alone are doomed to fail. Solving this conundrum is of paramount relevance for the design of antiAD clinical trials.

Here, we demonstrate that tau is not necessary for the $A \beta$ induced impairment of long-term synaptic plasticity and memory, or for amyloid deposition. Tau suppression can even unveil a deficit 
in basal neurotransmission in amyloid-depositing mice. The role of tau in $A \beta$-induced damage would be restricted only to the impairment of short-term synaptic plasticity and memory.

\section{Results}

Endogenous tau expression is not required for disruption of long-term synaptic plasticity and memory induced by overexpression of mutated $A P P$. To provide an in-depth analysis of the relationship between $A \beta$ and tau, we tested the effects of knocking out tau expression onto LTP reduction by mutated APP overexpression. To this end, we crossed Mapt-KO mice (32) with transgenic mice overexpressing human APP carrying the Swedish (APP KM670/671NL) and the Indiana (V717F) mutations (named TgAPP) (33). APP expression in these transgenics is driven by the neuron-specific prion promoter to generate a model of $\mathrm{AD}$-related amyloid pathology where $A \beta$ depositions are observed at 3-4 months of age (33). Interestingly, we found that basal synaptic transmission was impaired in 9- to 12-month-old TgAPP/Mapt-KO mice compared with TgAPP, Mapt-KO, and WT littermates (Figure 1A), suggesting that the combination of mutated APP overexpression with tau suppression is deleterious to basal neurotransmission. LTP analysis in slices from TgAPP/Mapt-KO and TgAPP mice revealed an impairment at 120 minutes after the theta-burst compared with WT or Mapt-KO littermates (Figure 1, B and C), whereas the impairment was not present in TgAPP/Mapt-KO mice at 30 minutes after tetanus (Figure 1, B and C). These findings show that tau suppression unveils a defect in basal neurotransmission in mice overexpressing mutated APP. Additionally, mutated APP overexpression with chronic expression and accumulation of naturally produced $A \beta$ affects long-term plasticity despite the absence of endogenous tau. The role of tau was confined to the short-term phase of LTP.

Given that LTP is a cellular correlate of memory, we evaluated cognitive function in TgAPP/Mapt-KO mice compared with the other groups. Analysis of spatial memory through the 2-day radial arm water maze (RAWM) test showed an impairment in TgAPP and TgAPP/Mapt-KO mice, which made more errors than their WT and Mapt-KO littermates (Figure 1D), suggesting decreased spatial memory in both animal models. Most importantly, tau suppression did not protect against the spatial memory damage in animals overexpressing mutated APP.

We obtained consistent results when we examined contextual fear memory after an electric shock. The amount of freezing in TgAPP and TgAPP/Mapt-KO mice was lower than in WT and Mapt-KO littermates when the animals were exposed to the same context at 24 hours after training (Figure 1E), suggesting that tau suppression does not protect against the impairment of contextual fear memory in mice overexpressing mutated APP.

Given that electrophysiological experiments showed that tau suppression protects against the damage of the initial phase of LTP in TgAPP/Mapt-KO mice, we wondered whether endogenous tau, although not required for the APP overexpression-induced impairment of long-term memory, blocks the effect of the overexpression onto short-term memory. To this end, we used fear conditioning that allows analyzing learning at specific time intervals after training. Evaluation of contextual fear learning at $30 \mathrm{~min}$ utes after training showed that TgAPP/Mapt-KO mice presented similar freezing as WT littermates (Figure 1F), suggesting that the short-term memory defect by mutated APP overexpression is rescued by tau suppression.

Analysis of amygdala-dependent cued memory at 24 hours after examination of contextual fear memory was also interesting. As previously shown $(34,35)$, it revealed an impairment of cued memory in TgAPP mice compared with WT mice. Interestingly, the defect was not rescued by tau suppression in TgAPP/Mapt-KO mice (Figure $1 G$ ), suggesting that tau suppression will not rescue the defect in emotional memory of AD patients.

No differences among genotypes were found in animal capability of perceiving the electric shock as measured in sensory threshold assessment (Supplemental Figure 1A; supplemental material available online with this article; https://doi.org/10.1172/ JCI137040DS1). Moreover, time and speed to reach a visible platform above the surface of the water (Supplemental Figure 1, B and C), and locomotor activity and anxiety-like behavior in an open field task (Supplemental Figure 1, D and E) were not affected, suggesting that differences among mice with different genotypes did not cause any sensorial, motor, or motivational defects that might produce the observed effects with the RAWM and contextual fear memory tests. Overall, these experiments suggest that tau suppression protects against the detrimental effects of $A \beta$ on synaptic function and memory only on short-term but not long-term memory.

We then analyzed amyloid load in TgAPP/Mapt-KO mice and TgAPP littermates. As previously shown on the J20 mice in a Mapt-KO background (1), this assessment did not reveal any difference between the 2 genotypes (Figure $1 \mathrm{H}$ ). This finding supports the observation that $A \beta$-induced synaptic plasticity and memory loss are independent of tau suppression. Most importantly, it extends to AD histopathology the concept that tau suppression is not beneficial against $\mathrm{A} \beta$-induced $\mathrm{AD}$ progression.

Endogenous tau expression is not required for disruption of longterm synaptic plasticity and memory induced by oA $\beta$ exposure. APP overexpression could affect neuronal function through a number of different mechanisms such as overproduction of APP itself or different fragments of its processing, including $A \beta$. Thus, to further investigate the relationship between $A \beta$ and tau and determine the role of $A \beta$ in the observed long-term synaptic plasticity and memory defects, we studied LTP following oA $\beta$ administration (Figure 2A) in brain slices from 4- to 6-month-old Mapt-KO mice. In preliminary experiments, we compared basal synaptic transmission in slices from Mapt-KO mice and WT littermates and found a similar input/output relationship (Figure 2B). Next, we confirmed previous findings (36) showing that a brief, 20-minute perfusion with a preparation containing synthetic oA $\beta(200 \mathrm{nM})$ before a theta-burst stimulation impairs hippocampal LTP at the CA3-CA1 synapse in slices from WT mice both at a short time after induction of potentiation (30 minutes) and at a later time point (120 minutes) (Figure 2, C and D). However, oA $\beta$ behaved differently on slices from Mapt-KO mice. It was able to impair the late phase of LTP at 120 minutes after the theta burst, whereas no impairment was present at 30 minutes after the tetanus (Figure 2, $\mathrm{C}$ and D). Importantly, application of oA $\beta$ did not affect basal neurotransmission, as shown by lack of drifting of the baseline (Figure 2C) and similar input/output relationship in slices from Mapt-KO and WT mice treated with either vehicle or oA $\beta$ (Supplemental 

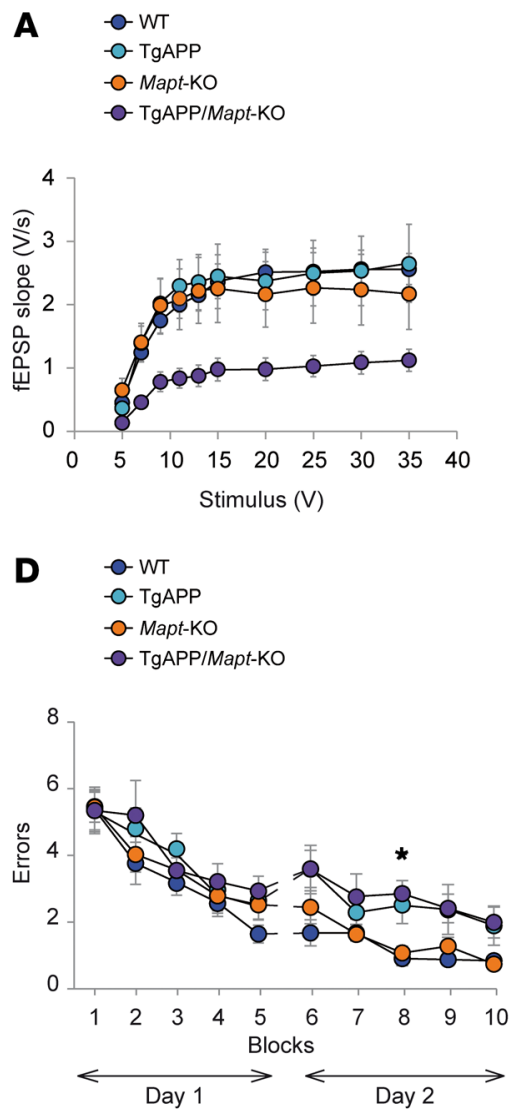

B
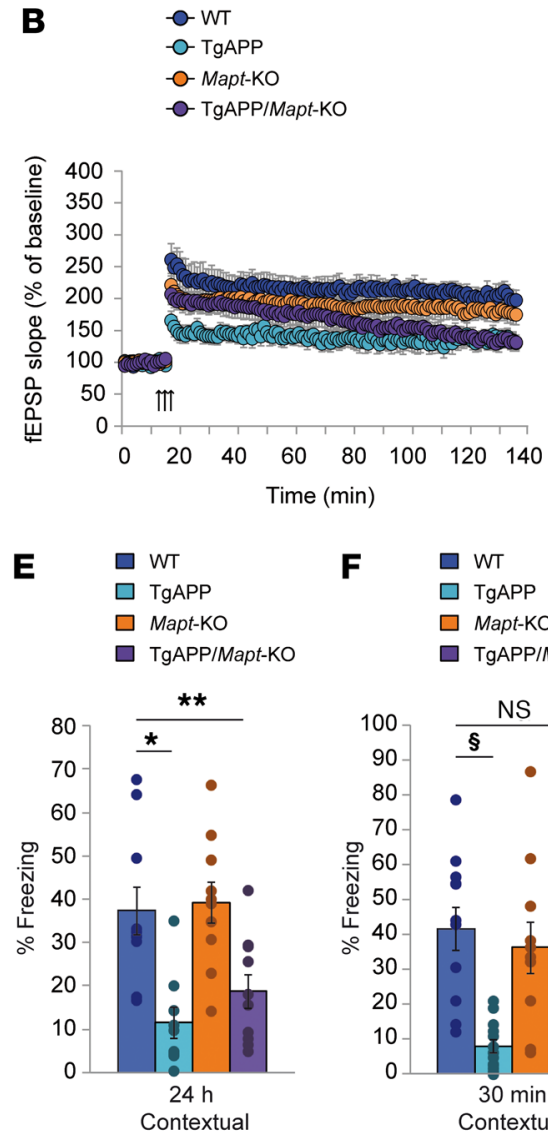
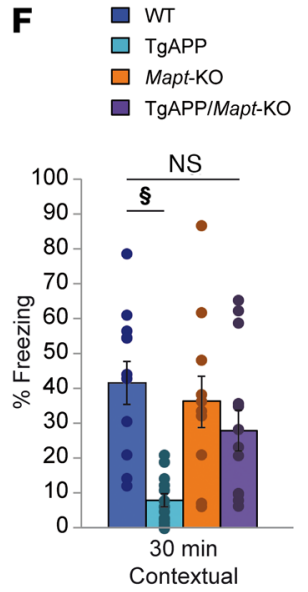
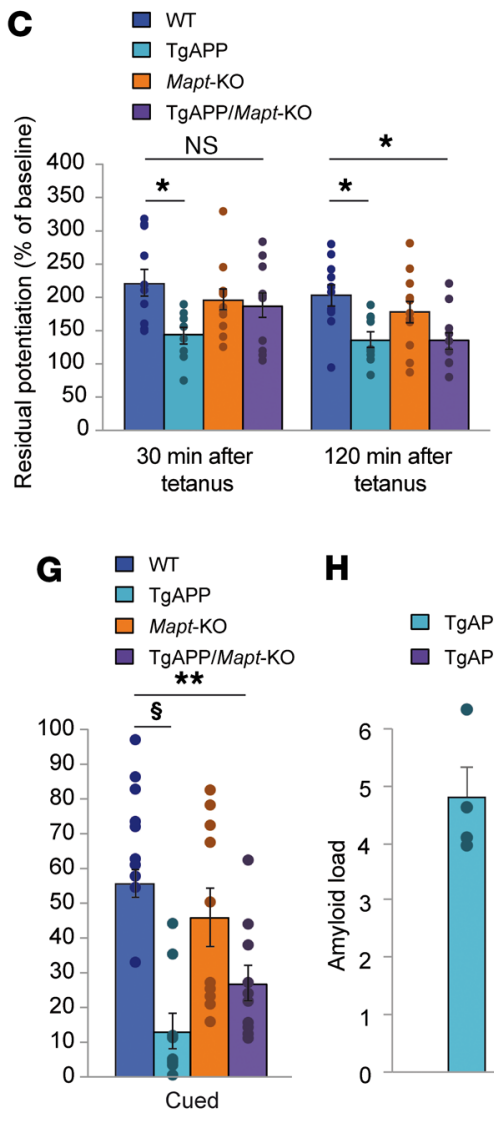

H

$$
\square \text { TgAPP }
$$

$\square$ TgAPPIMapt-KO

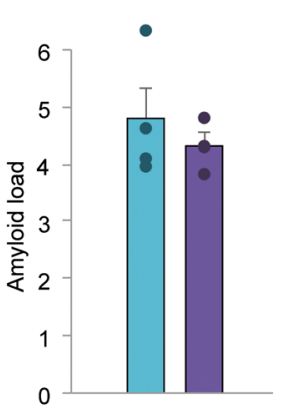

Figure 1. Mutated APP overexpression impairs long-term but not short-term synaptic plasticity and memory in Mapt-KO mice. (A) Basal neurotransmission is normal in Mapt-KO and TgAPP slices (ANOVA for repeated measures $\mathrm{F}_{(2.26)}=1.639, P=0.108$ ), but impaired in TgAPP/Mapt-KO slices (ANOVA for repeated measures $F_{(1,17)}=31.106, P<0.0001 ; n=9 \mathrm{WT}, n=9$ TgAPP, $\left.n=12 \mathrm{Mapt}-\mathrm{KO}, n=11 \mathrm{TgAPP} / \mathrm{Mapt}-\mathrm{KO}\right)$. (B) Endogenous tau suppression does not protect TgAPP slices against LTP impairment (ANOVA for repeated measures $\mathrm{F}_{(1.18)}=6.085, P=0.01$, WT vs. TgAPP; ANOVA for repeated measures $\mathrm{F}_{(1,21)}=5.119, P<0.05$, WT vs. TgAPP/Mapt-KO; $n=11 \mathrm{WT}, n=9$ TgAPP, $\left.n=12 \mathrm{Mapt}-\mathrm{KO}, n=11 \mathrm{TgAPP} / \mathrm{Mapt}-\mathrm{KO}\right)$. (C) Analysis of slices displayed in B shows normal LTP at 30 minutes after tetanus in TgAPP/Mapt-KO slices (1-way ANOVA with Bonferroni correction $P=1$, WT vs. TgAPP/Mapt-KO), but not at 120 minutes $(P<0.05)$. (D) RAWM performance is impaired in TgAPP and TgAPP/Mapt $-\mathrm{KO}$ mice (ANOVA for repeated measures, day $2 \mathrm{~F}_{(3,39)}=5.961, P=$ 0.002 ; 1-way ANOVA with Bonferroni correction $P<0.05$ in WT vs. TgAPP and $P=0.005$ vs. TgAPP/Mapt-KO for block $8 ; n=10$ WT, $n=11$ TgAPP, $n=10$ Mapt-KO, $n=12$ TgAPP/Mapt-KO). (E) Contextual fear memory is impaired in TgAPP and TgAPP/Mapt-KO mice at 24 hours after training (1-way ANOVA, $F_{(3,35)}=8.897, P<0.0001$; Bonferroni's $P<0.005$, WT vs. TgAPP; $P<0.05$, WT vs. TgAPP/Mapt $-\mathrm{KO}$ mice; $n=10 \mathrm{WT}, n=9$ TgAPP, $n=10$ Mapt-KO, $n=10$ TgAPP/Mapt-KO). (F) Endogenous tau suppression protects TgAPP mice against short-term contextual fear memory impairment (1-way ANOVA with Bonferroni correction $P=0.472$, WT vs. TgAPP/Mapt-KO mice; $n=11 \mathrm{WT}, n=13$ TgAPP, $n=10$ Mapt-KO, $n=13$ TgAPP/Mapt-KO). (C) Cued fear memory is impaired in TgAPP and TgAPP/Mapt-KO mice (1-way ANOVA with Bonferroni correction $\mathrm{F}_{(3,35)}=10.207, P<0.0001$; Bonferroni's $P<0.01$ for both genotypes vs. WT). (H) Endogenous tau suppression does not influence amyloid load in TgAPP mice (2-sample unpaired $t$ test, $t_{(6)}=0.766 P>0.05 ; n=4$ for both groups). ${ }^{*} P<0.05 ;{ }^{* *} P<0.01 ;{ }^{\circledR} P<0.0001$.

Figure 2A). These findings strongly support the hypothesis that the role of endogenous tau in A $\beta$-induced impairment of LTP is confined to the early phase without affecting the late phase of LTP.

We then investigated the effects of tau suppression on oA $\beta$ induced memory loss in Mapt-KO mice. As previously demonstrated (19), a brief, 60-second infusion of a preparation containing synthetic oA $\beta$ into dorsal hippocampi ( $200 \mathrm{nM}$, in $1 \mu \mathrm{L}$ bilaterally, 20 minutes before the first and seventh trial on both days of the 2-day RAWM task) increased the number of errors with the 2-day RAWM in both WT and Mapt-KO mice compared with vehicle-treated littermates (Figure 2E), suggesting decreased spatial memory after $\mathrm{A} \beta$ treatment regardless of endogenous tau suppression.

We obtained consistent results when we bilaterally infused the same preparation (200 nM over 60 seconds) into the hip- pocampi 20 minutes before the electric shock to induce fear conditioning. The amount of freezing in oA $\beta$-treated WT and Mapt-KO littermates was lower than in vehicle-treated siblings when the animals were exposed to the same context at 24 hours after training (Figure $2 \mathrm{~F}$ ), suggesting an impairment of contextual fear memory. Thus, endogenous tau is not needed for oA $\beta$ to impair long-term memory.

Electrophysiological experiments showed that tau suppression protects against oA $\beta$ damage of the initial phase of LTP. Thus, we wondered whether endogenous tau, although not required for the oA $\beta$-induced impairment of long-term memory, could block the effect of oA $\beta$ onto short-term memory. Evaluation of contextual fear learning at 30 minutes after training showed that Mapt-KO mice infused with $200 \mathrm{nM}$ oA $\beta$ at 20 minutes before training 


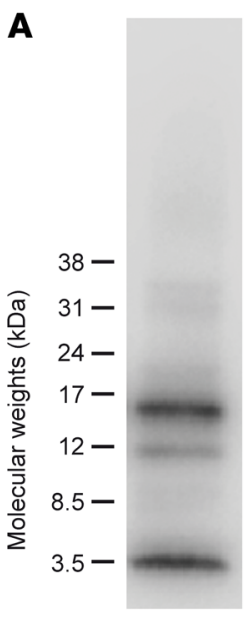

B

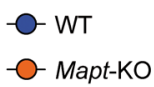

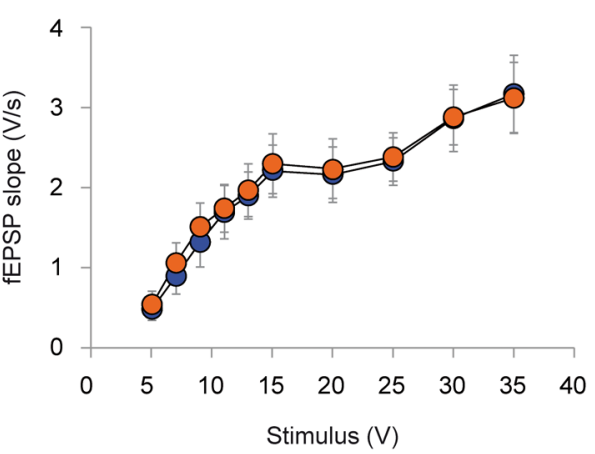

C
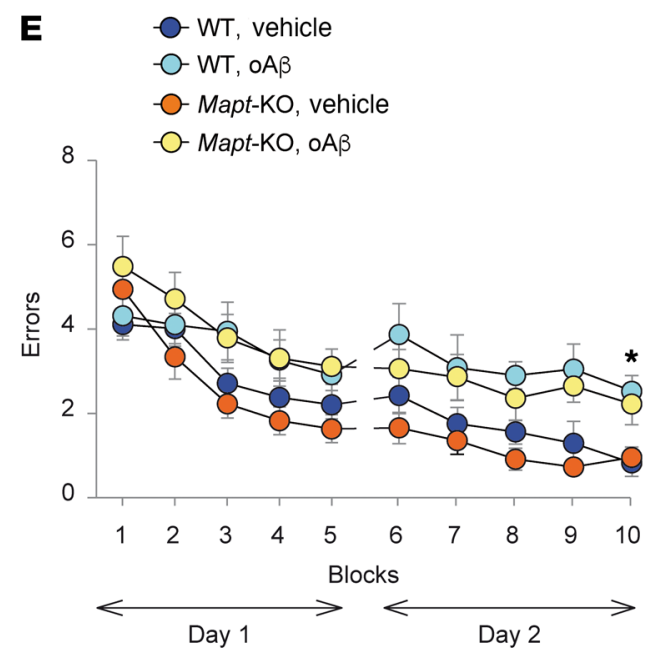

G
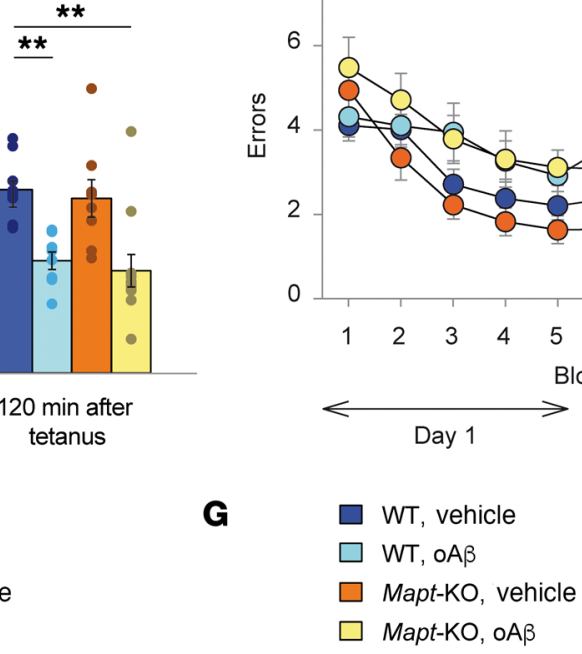

120 min after

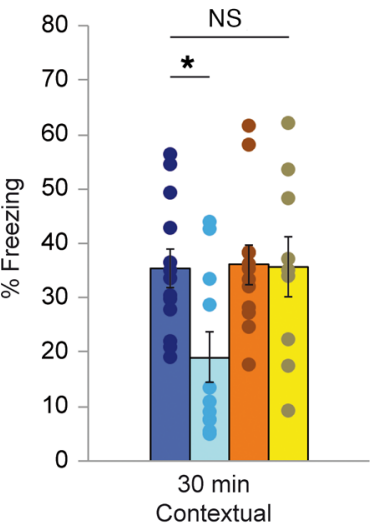

Contextual

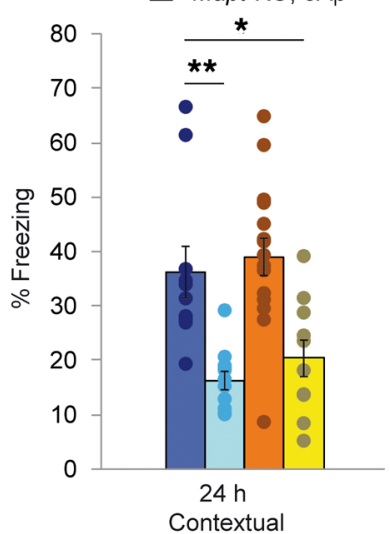

$\mathbf{F}$

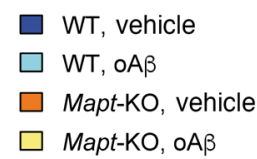

Contextual
- WT, vehicle

$-O-W T, o A B$

- Mapt-KO, vehicle

- - Mapt-KO, oA $\beta$

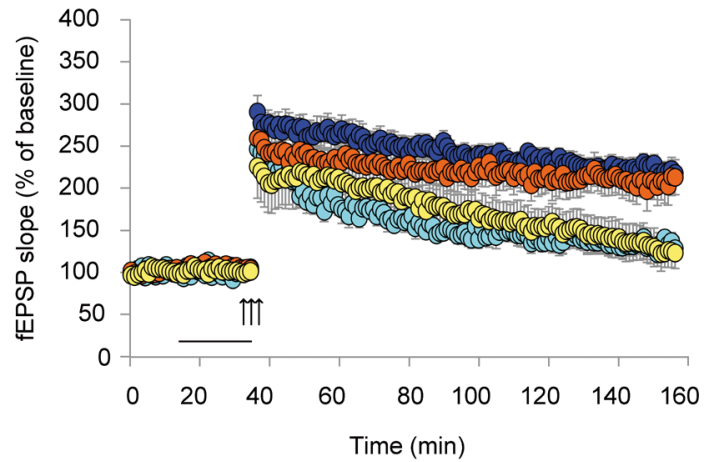
H
$\square$ WT, vehicle
$\square$ WT, OAB
$\square$ Mapt-KO, vehicle
$\square$ Mapt-KO, OAß

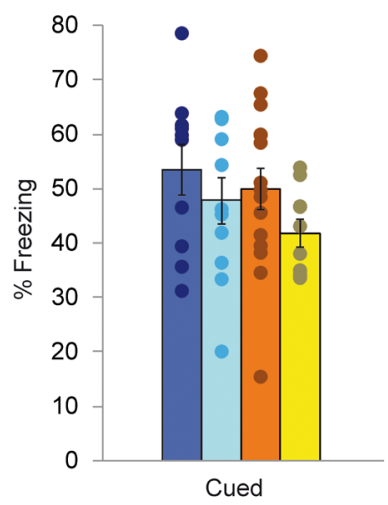


Figure 2. Extracellular oA $\beta$ impairs long-term but not short-term synaptic plasticity and memory in Mapt-KO mice. (A) Tris-Tricine SDS-PAGE Western blotting of $A \beta_{42}$ samples (prepared in nondenaturing/nonreducing conditions before loading) showing different bands corresponding to monomers, and oligomers. (B) Basal neurotransmission is similar in WT and Mapt-KO slices ( $n=18 / 17$; ANOVA for repeated measures $\mathrm{F}_{(1,33)}=0.031$, $P=0.861)$. (C) LTP is similar in WT and Mapt-KO slices $(n=10 / 8$; ANOVA for repeated measures $\left.F_{(1,16)}=1.176, P=0.294\right)$. oA $\beta(200 \mathrm{nM})$ treatment impairs LTP in WT (ANOVA for repeated measures $F_{(1,16)}=31.192, P<$ $0.0001 ; n=8$ ) and Mapt-KO (ANOVA for repeated measures $F_{(1,19)}=6.219$, $P<0.05 ; n=8$ ) slices. (D) Analysis of slices displayed in $\mathbf{C}$ shows protection against LTP impairment at 30 minutes after tetanus in Mapt-KO+oA $\beta$ slices (1-way ANOVA with Bonferroni correction $P=0.282$ in WT+vehicle vs. Mapt-KO+oA $\beta)$, but not at 120 minutes $(P=0.001)$. (E) oA $\beta(200 \mathrm{nM})$ impairs RAWM performance in WT and Mapt-KO mice (day 2 ANOVA for repeated measures $\mathrm{F}_{(3.36)}=5.598, P<0.005$; 1-way ANOVA with Bonferroni correction $P<0.05 \mathrm{WT}+\mathrm{OA} \beta$ and Mapt-KO+oA $\beta$ for block 10; $n=10$ $\mathrm{WT}+$ vehicle and Mapt-KO+oA $\beta, n=11 \mathrm{WT}+\mathrm{OA} \beta, n=9 \mathrm{Mapt}-\mathrm{KO}+$ vehicle). (F) Contextual fear memory is impaired in WT and Mapt-KO mice infused with $o A \beta$ tested at 24 hours after training (1-way ANOVA with Bonferroni correction $\mathrm{F}_{(3.42)}=10.836, P<0.0001$; Bonferroni's $P<0.005$ in WT+vehicle vs. WT+oA $\beta ; P<0.05$ in WT+vehicle vs. Mapt $-K 0+0 A \beta ; n=10 \mathrm{WT}+$ vehicle, $n=11 \mathrm{WT}+\mathrm{oA} \beta, n=15 \mathrm{Mapt}-\mathrm{KO}+$ vehicle, $n=10 \mathrm{Mapt}-\mathrm{KO}+\mathrm{oA} \beta)$. (C) Endogenous tau suppression protects against oA $\beta$-induced impairment of shortterm contextual fear memory at 30 minutes after training (1-way ANOVA with Bonferroni correction $\mathrm{F}_{(3.41)}=3.778, P<0.05$; Bonferroni's $P<0.05$ in WT+vehicle vs. WT+oA $\beta ; P=1$ in WT+vehicle vs. Mapt-KO+oA $\beta ; n=13$ WT+vehicle, $n=11 \mathrm{WT}+o A \beta, n=12 \mathrm{Mapt}-\mathrm{KO}+$ vehicle, $n=9$ Mapt-KO+oA $\beta$ ). (H) No differences were detected among WT and Mapt-KO mice treated with vehicle or $O A \beta$ in cued conditioning test (1-way ANOVA $F_{(3,42)}=1.347$, $P=0.272) .{ }^{*} P<0.05 ;{ }^{* *} P<0.01$

did not exhibit a significant reduction of freezing compared with vehicle-treated WT mice (Figure $2 \mathrm{G}$ ), suggesting a protection against $\mathrm{A} \beta$-induced short-term memory loss in Mapt-KO mice.

The A $\beta$-induced defects observed with the RAWM and contextual fear conditioning could be attributed to hippocampal impairment because in control experiments we did not find any differences in cued memory among the 4 groups of mice (Figure $2 \mathrm{H})$, suggesting no amygdala involvement in the effects of $A \beta$ in fear memory. Moreover, oA $\beta$ did not modify animal capability of perceiving the electric shock as measured in sensory threshold assessment (Supplemental Figure 2B), time, and speed to reach a visible platform above the surface of the water (Supplemental Figure 2, C and D), or locomotor activity and anxiety-like behavior in an open field task (Supplemental Figure 2, E and F), suggesting that $\mathrm{A} \beta$ did not cause any sensorial, motor, or motivation defects that might have been responsible for its effects on RAWM and contextual fear memory tests.

Exogenously applied oTau impairs long-term synaptic plasticity and memory regardless of endogenous tau suppression. Similar to oA $\beta$ (23), oTau impairs both hippocampal LTP and memory (10, 22, 23). Both peptides share APP as a molecule necessary to reduce LTP and memory (19). We therefore investigated the relationship between tau oligomers (Figure 3A) and tau itself with respect to the impairment of LTP and memory, by supplementing synapses with oTau in the absence of endogenous tau. Recording of basal synaptic transmission in slices from 4- to 6-month-old Mapt-KO and WT littermate mice confirmed the lack of differences between the 2 groups shown in Figure 1A and Figure 2B (Figure 3B). Slices perfused for 20 minutes with $50 \mathrm{nM}$ oTau before inducing LTP revealed a marked reduction of potentiation at 120 minutes after the tetanus in slices from Mapt-KO and WT mice compared with vehicle-treated slices (Figure 3, C and D). Conversely, no differences between oTau- and vehicle-treated slices were observed when analyzing the initial phase of LTP in slices from Mapt-KO mice (Figure 3, C and D). In addition, oTau did not affect basal neurotransmission, as shown by lack of drifting of the baseline (Figure 3C) and similar input/output relationship in slices from Mapt-KO and WT mice treated with either vehicle or oTau (Supplemental Figure 3A). Collectively, these results suggest that endogenous tau is not needed for the oTau-induced impairment of the late phase of LTP whereas it is needed in the initial phase of LTP.

To examine the relevance of human tau to memory regardless of suppression of endogenous murine tau, we administered oTau through cannulas into the dorsal hippocampi $(500 \mathrm{nM}$, in $1 \mu \mathrm{L}$ bilaterally, 20 and 180 minutes before the first trial of both days of the 2-day RAWM task, over 60 seconds). Infusion of oTau revealed a higher number of errors both in the WT and Mapt-KO mice (Figure 3E). Moreover, tau suppression did not protect mice against the damage of contextual fear memory induced by oTau (500 nM, bilaterally, 20 and 180 minutes before the electric shock, over 60 seconds) at 24 hours after the electric shock (Figure $3 F)$. Similar to oA $\beta$, the protection was instead present when memory was assessed at 30 minutes (Figure 3G). Finally, we did not observe any behavioral differences among various groups of mice when they were tested for cued conditioning (Figure 3H), sensory threshold (Supplemental Figure 3B), visible platform (Supplemental Figure 3, C and D), and open field (Supplemental Figure 3, E and F). Taken together, these data demonstrate that similar to $\mathrm{oA} \beta$, tau suppression does not protect against the detrimental effects of oTau on long-term synaptic plasticity and memory.

Blockage of soluble guanylyl cyclase (sGC) abolishes the protective effect of tau suppression against oA $\beta$ - or oTau-induced defect in shortterm synaptic plasticity. The early phase of LTP depends on the nitric oxide/cGMP signaling pathway (37). For instance, $1 H$-[1,2,4] oxadiazolo[4,3- $\alpha$ ]quinoxalin-1-one (ODQ), an inhibitor of sGC, the enzyme that produces cGMP, reduces the early phase of LTP $(38,39)$. To provide insight into the molecular mechanism by which loss of tau confers protection against reduction in short-term plasticity, we examined whether inhibiting sGC blocks the rescue of short-term plasticity by tau suppression. Basal synaptic transmission was similar in slices from 4- to 6-month-old Mapt-KO and WT littermate mice (Figure 4A), confirming observations shown in Figure $1 \mathrm{~A}$, Figure $2 \mathrm{~B}$, and Figure $3 \mathrm{~B}$. As previously demonstrated (40), ODQ perfusion $(10 \mu \mathrm{M}$, for 10 minutes before the theta-burst) dramatically reduced LTP in WT slices (Figure 4B). A similar reduction in potentiation was present in Mapt-KO slices treated with the inhibitor (Figure 4C). As shown in Figure 2, C and D, and Figure 3, $\mathrm{C}$ and $\mathrm{D}$, both oA $\beta$ and oTau were not capable of impairing the early phase of LTP in Mapt-KO slices (Figure 4C). However, ODQ perfusion unveiled a defect of LTP at 30 minutes after the tetanus in Mapt-KO slices treated with either oA $\beta$ or oTau (Figure 4, C and D). Finally, ODQ did not further depress LTP in oA $\beta$ - or oTau-treated WT slices (Figure 4, B, D, and E). The above findings suggest that disruption of CGMP signaling reverses the neuroprotective action of endogenous tau suppression against oA $\beta$ - or oTau-induced impairments of the early phase of synaptic plasticity. 

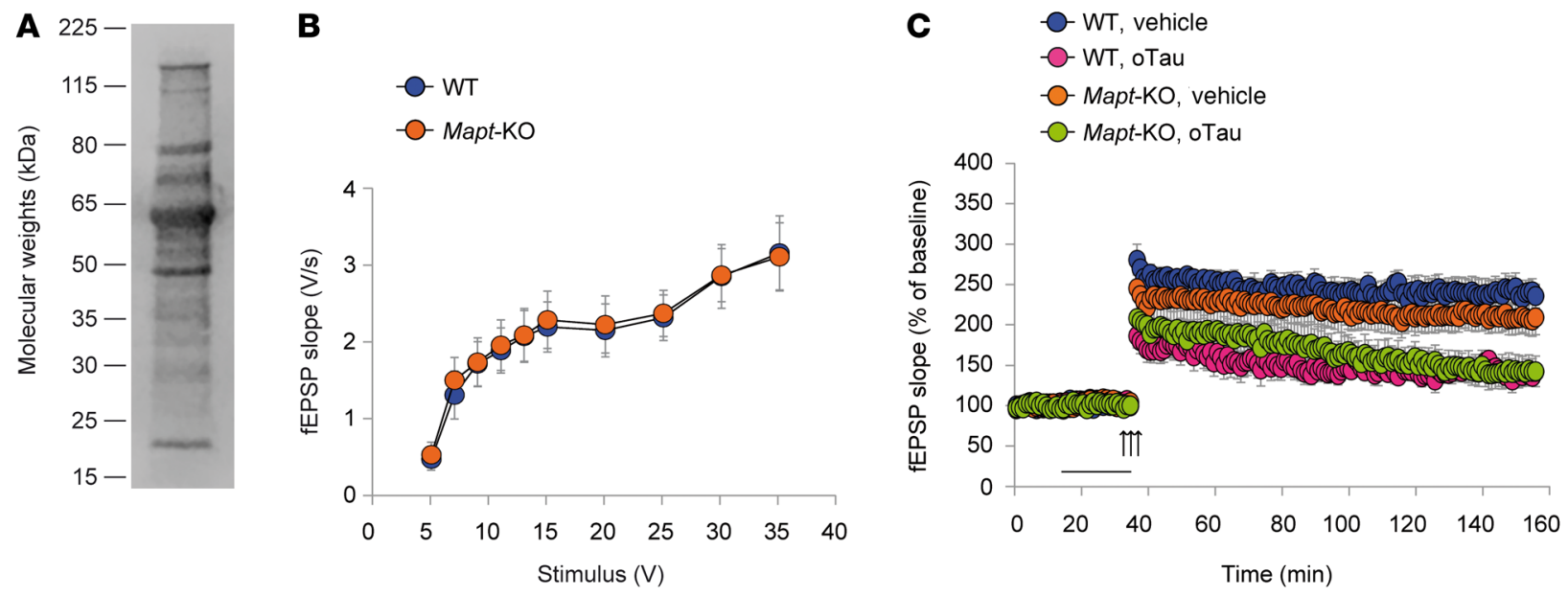
D $\square W T$, vehicle
$\square$ WT, oTau
$\square$ Mapt-KO, vehicle

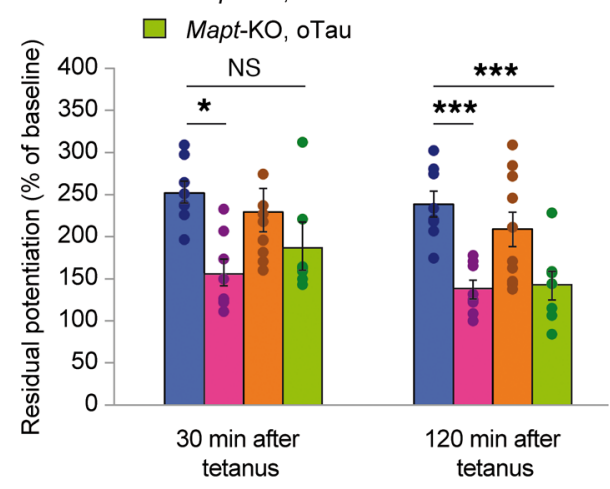

E

- WT, vehicle

- WT, oTau

-O-Mapt-KO, vehicle
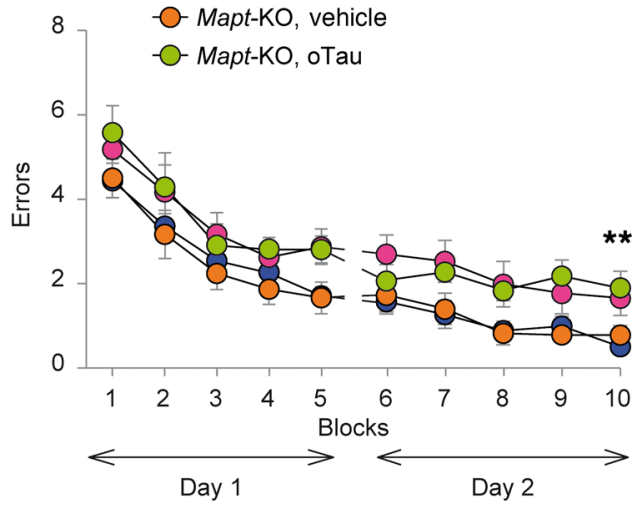

F
$\square \mathrm{WT}$, vehicle
$\square$ WT, oTau
$\square$ Mapt-KO, vehicle
$\square$ Mapt-KO, oTau

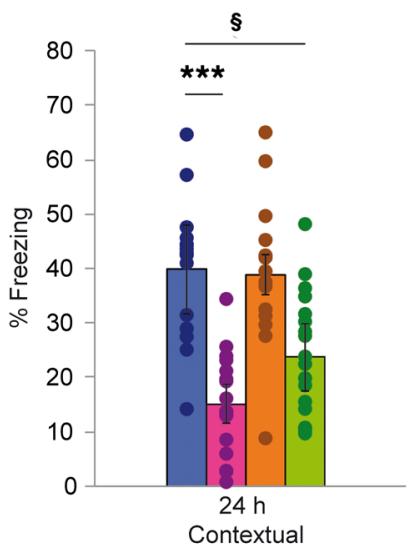

G
H
$\square$ WT, vehicle
$\square$ WT, oTau
$\square$ Mapt-KO, vehicle
$\square$ Mapt-KO, oTau

$\square$ Mapt-KO, vehicle

$\square$ Mapt-KO, oTau

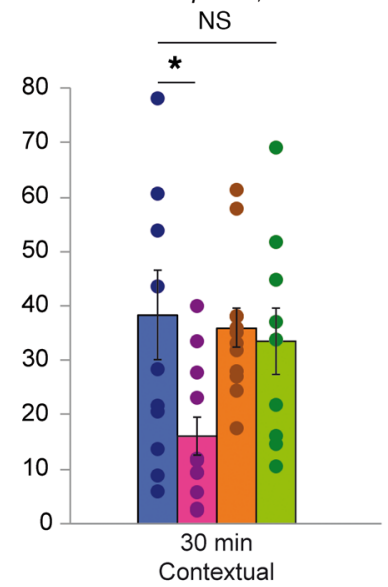

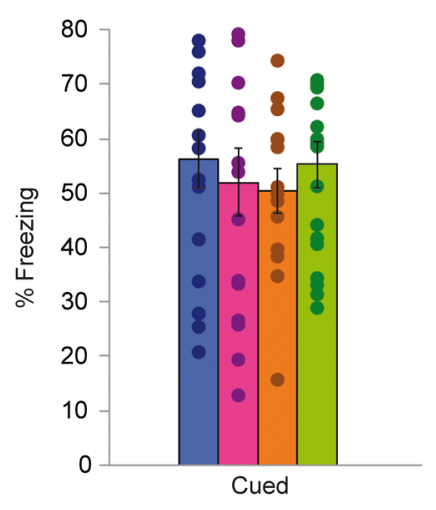


Figure 3. Extracellular oTau impairs long-term but not short-term synaptic plasticity and memory in Mapt-KO mice. (A) Immunoblot for recombinant tau oligomers using anti-tau antibody after isolation and oligomerization. (B) Basal neurotransmission is similar in WT and Mapt-KO slices (ANOVA for repeated measures $\mathrm{F}_{(1,33)}=0.017, P=0.897$; $n=18 / 15$ ). (C) oTau (50 nM) impairs LTP in WT and Mapt-KO slices (ANOVA for repeated measures $F_{(1,14)}=27.77, P<0.0001$ in WT+vehicle vs. WT+oTau; $\mathrm{F}_{(1,13)}=9.44, P<0.01$ in WT+vehicle vs. Mapt-KO+oTau; $n=8 \mathrm{WT}$ +vehicle, $n=10 \mathrm{WT}+\mathrm{oTau}, n=7 \mathrm{Mapt}-\mathrm{KO}+$ vehicle, $n=8 \mathrm{Mapt}-\mathrm{KO}+\mathrm{oTau})$. (D) Analysis of slices displayed in $\mathbf{C}$ shows protection against LTP impairment at 30 minutes after tetanus in Mapt-KO+oTau slices (1-way ANOVA with Bonferroni correction $P=0.369$ in WT+vehicle vs. Mapt-KO+oTau), but not at 120 minutes $(P=0.002)$. (E) oTau $(500 \mathrm{nM})$ impairs RAWM performance in WT and Mapt-KO mice (day 2 ANOVA for repeated measures $F_{(3,32)}=5.431, P<$ 0.005 ; 1-way ANOVA with Bonferroni correction $P<0.05$ in WT+vehicle vs. WT+oTau; $P=0.005$ in WT+vehicle vs. Mapt-K0+oTau for block 10; $n=13$ WT+vehicle, $n=8 \mathrm{WT}+\mathrm{oTau}, n=8 \mathrm{Mapt}-\mathrm{KO}+\mathrm{vehicle,} n=7 \mathrm{Mapt}-\mathrm{KO}+\mathrm{oTau}$ ) (F) Contextual fear memory is impaired in WT and Mapt-KO mice infused with oTau tested at 24 hours after training (1-way ANOVA with Bonferroni correction $\mathrm{F}_{(3,60)}=16.541, P<0.0001$; Bonferroni's $P<0.0001$ in WT+vehicle vs. WT+oTau; $P=0.001$ in WT+vehicle vs. Mapt-KO+oTau; $n=16 \mathrm{WT}+$ vehicle, $n=16$ WT+oTau, $n=14$ Mapt-KO+vehicle, $n=18$ Mapt-KO+oTau). (C) Endogenous tau suppression protects against the oTau-induced impairment of short-term contextual fear memory (1-way ANOVA with Bonferroni correction $\mathrm{F}_{(3,40)}=3.463, P<0.05$; Bonferroni's $P<0.05$ in WT+vehicle vs. WT+oTau; $P=1$ in WT+vehicle vs. Mapt-KO+oTau; $n=11 \mathrm{WT}+\mathrm{ve}-$ hicle, $n=12 \mathrm{WT}+\mathrm{oTau}, n=12 \mathrm{Mapt}-\mathrm{KO}+$ vehicle, $n=9 \mathrm{Mapt}-\mathrm{KO}+\mathrm{OTau})$. (H) Cued fear memory is similar in WT and Mapt-KO mice treated with vehicle or oTau (1-way ANOVA $\mathrm{F}_{(3,60)}=0.269, P=0.847$ ). ${ }^{*} P<0.05$; ${ }^{* *} P<0.01$; ${ }^{* *} P$ $<0.005 ; \$ P<0.0001$.

Overexpression of WT human tau abolishes the protective effect of tau suppression against oA $\beta$-induced short-term defects in LTP and memory. The protection against the negative effects of $\mathrm{A} \beta$ onto the initial phase of LTP and short-term memory in Mapt-KO mice might not be specific to altered tau expression. To determine specificity of the effect, we overexpressed human WT $4 \mathrm{R} / 2 \mathrm{~N}$ tau in Mapt-KO mice by generating htau/Mapt-KO mice. The mice express WT, full-length oligomer prone human tau (2N4R htau) using the prion cos-tet promoter, which results in a largely neuronal expression of the transgene (ref. 41, Figure 5A, and Supplemental Figure 4A). These mice display human oTau at 8 months of age (Figure 5B) when TOC1-positive oTau levels were equal to approximately $0.5 \mathrm{nM}$, and both LTP and memory impairments were not yet present given that they appeared only after 10 months of age (Supplemental Figure 4, B-F). Analysis of basal synaptic transmission at 6 to 8 months confirmed normal neurotransmission in slices from htau/Mapt-KO compared with those derived from both Mapt-KO and WT littermates (Figure 5C). Moreover, LTP was normal compared with Mapt-KO and WT littermates (Figure 5D). However, when slices from htau/Mapt-KO were perfused with subtoxic doses $(50 \mathrm{nM})$ of oA $\beta$ before the tetanus, they exhibited reduced LTP both at 30 minutes and 120 minutes after the tetanus, whereas this concentration of $A \beta$ was not sufficient to disrupt plasticity in slices from WT or Mapt-KO littermates (Figure 5 , D and $\mathrm{E}$ ), presumably because $50 \mathrm{nM} \mathrm{A} \beta$ concentration is subthreshold for LTP impairment $(42,43)$.

Likewise, an oA $\beta$ concentration subthreshold for memory impairment, $75 \mathrm{nM}$ (42), impaired spatial memory (Figure 5F) and contextual fear learning in htau/Mapt-KO mice but not in Mapt-KO and WT mice (Figure 5, G and H). The impairment of contextual memory was present both at 30 minutes and 24 hours after the electric shock (Figure 5, G and H). No differences were found in cued conditioning (Figure 5I), sensory threshold (Supplemental Figure 5A), visible platform (Supplemental Figure 5, B and C), and open field (Supplemental Figure 5, D and E) among groups of mice. Overall, these experiments demonstrate that the protective effect of tau suppression against the $A \beta$-induced reduction of the initial phase of LTP and short-term memory is specific to tau.

\section{Discussion}

The prevailing hypothesis in $\mathrm{AD}$ research is that $\mathrm{A} \beta$ precedes tau in causing pathology. Tau would mediate, or accelerate, the pathogenic effects of $A \beta$ (44). Such a hierarchical profile in the chain of events leading to memory loss in $\mathrm{AD}$ is used as an explanation for the failures of many clinical trials, mostly targeting A $\beta$. Two types of strategies are currently being implemented to overcome this obstacle. In one line of research, anti-A $\beta$ therapies are being administered before the overt disease manifestation. In the other, various aspects of tau pathology, including tau posttranslational modifications, tau levels, and tau aggregation status, are the main target of tau-tailored therapies. However, this model was recently challenged by studies suggesting that $A \beta$ and tau act in parallel instead of being in a series $(10,19,24,25)$. In this manuscript, we demonstrate that neither exogenous oA $\beta$ nor oTau need endogenous mouse tau to negatively impact the late phase of CA3-CA1 LTP and long-term hippocampal memory. Moreover, we find that tau suppression does not reduce amyloid load in a mouse model of amyloid deposition, and even unravels a defect in basal neurotransmission in the model. These evidences suggest that, at least for certain electrophysiological, behavioral, and histopathological aspects, $A \beta$ and tau act in parallel, and not in a series as the amyloid cascade hypothesis predicts.

Tau suppression was shown to protect against synaptic plasticity and memory defects in transgenic mice overexpressing mutated forms of $\operatorname{APP}(1,45,46)$. However, we found that TgAPP/Mapt-KO mice display abnormal synaptic plasticity and memory. Different results between our experiments and earlier investigations might reflect the different experimental paradigm used among studies. For instance, previous investigations examined LTP at the medial perforant path synapse with the dentate gyrus and followed it for 60 minutes (46). In contrast, we have investigated the CA3-CA1 synapse for 2 hours. Additionally, previous studies used either the Morris water maze or the T-maze $(1,45,46)$, whereas we used the RAWM and fear conditioning. Additionally, different APP and tau-KO models were used. For APP models, the CRND8 mouse was used in our experiments, unlike the APP23 and the J20 mouse in the other studies. For tau-KO models, the Jackson 007251 strain (32) in which the mouse tau gene was functionally disrupted by replacing exon 1 with the neomycin resistance cassette was used in our experiments and Roberson's experiments (46), whereas models in which tau expression was disrupted through insertion of the EGFP cDNA into exon 1 of the tau locus Mapt were used in the Ittner studies (45). Regardless, these results strongly support the hypothesis that the tau dependence for the effects of oA $\beta$ is confined to certain aspects of the pathology, but not necessarily to all aspects of the disease including the late phase of LTP, long-term memory, basal neurotransmission, or amyloid deposition. Consistent with this conclu- 
A

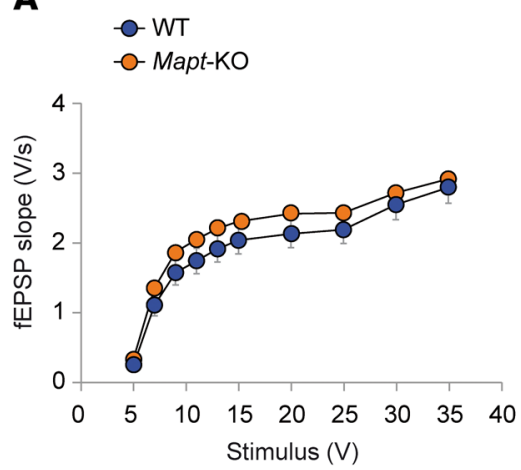

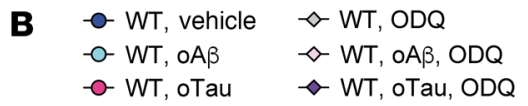

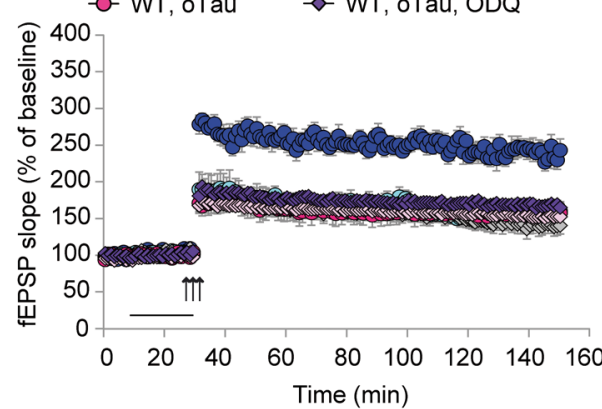

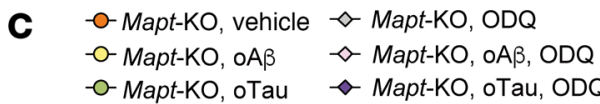

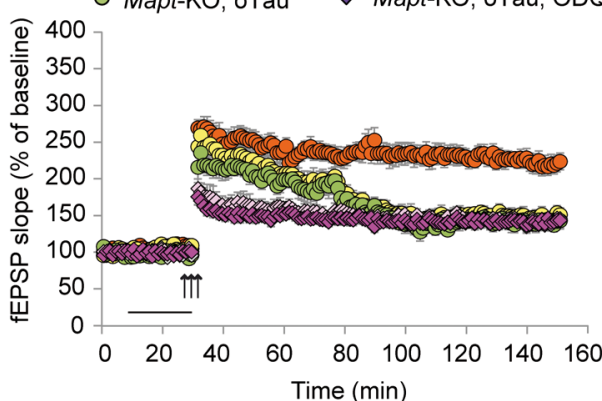

D

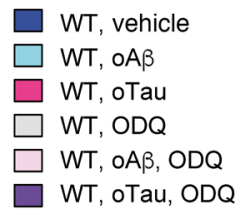

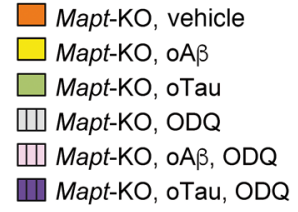

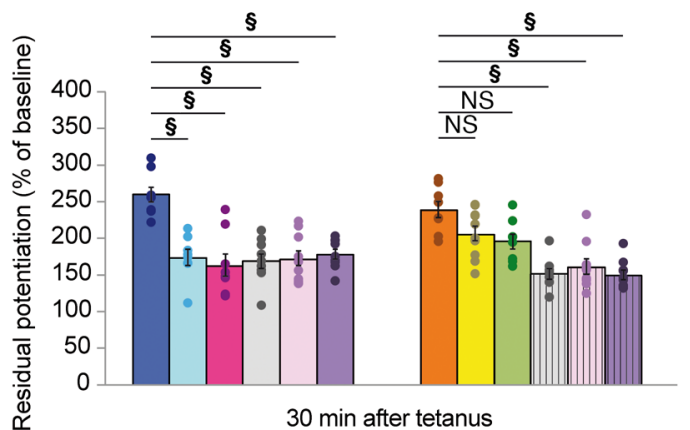

E

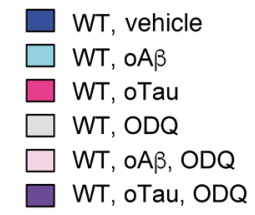

Mapt-KO, vehicle

$\square$ Mapt-KO, oA $\beta$

$\square$ Mapt-KO, oTau

W Mapt-KO, ODQ

Ш Mapt-KO, OAß, ODQ

W Mapt-KO, oTau, ODQ

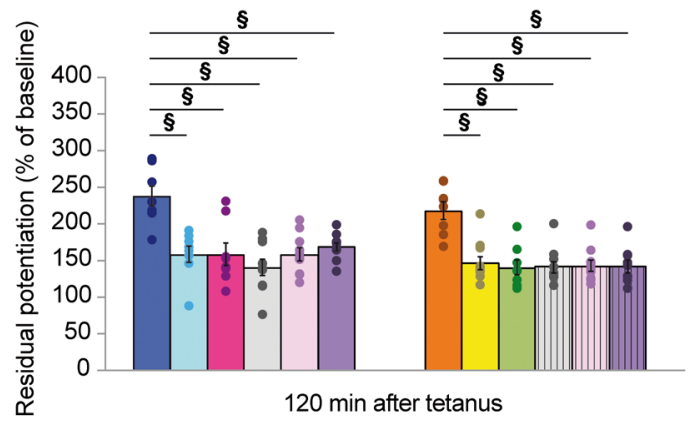

Figure 4. Inhibition of SGC abolishes the neuroprotective effect of tau suppression against oA $\beta$ - or oTau-induced impairments in short-term plasticity. (A) Basal neurotransmission is similar in WT and Mapt-KO slices $\left(n=53 / 54\right.$; ANOVA for repeated measures $\left.F_{(1,105)}=0.977, P=0.325\right)$. (B) Application of either oA $\beta(200 \mathrm{nM})$, oTau $(50 \mathrm{nM})$, ODQ $(10 \mu \mathrm{M})$, oA $\beta+$ ODQ, or oTau+ODQ impairs LTP in WT slices (ANOVA for repeated measures $F_{(1,14)}=38.46, P<0.0001$ in WT+vehicle vs. WT+oA $\beta$; ANOVA for repeated measures $F_{(1,14)}=28.76, P<0.0001$ in WT+vehicle vs. WT+oTau; $F_{(1,15)}=49.97, P<0.0001$ in WT+vehicle vs. WT+ODQ; $F_{(1,16)}=42.90, P<0.0001$ in WT+vehicle vs. WT+oA $\beta+O D Q ; F_{(1,16)}=65.02, P<0.0001$ in WT+vehicle vs. WT+oTau+ODQ; $n=8$ WT+vehicle, $n=8$ $\mathrm{WT}+\mathrm{OA} \beta, n=8 \mathrm{WT}+\mathrm{oTau}, n=9 \mathrm{WT}+\mathrm{ODQ}, n=10 \mathrm{WT}+\mathrm{OA} \beta+\mathrm{ODQ}, n=10 \mathrm{WT}+\mathrm{OTau}+\mathrm{ODQ}$ ). (C) Application of either oA $\beta, \mathrm{OTau}, \mathrm{ODQ}, \mathrm{oA} \beta+\mathrm{ODQ}, \mathrm{or}$ oTau+ODQ impairs LTP in Mapt-KO slices $\left(F_{(116)}=18.99, P<0.0001\right.$ in Mapt-KO+vehicle vs. Mapt-KO+oA $\beta ; F_{(114)}=25.25, P<0.0001$ in Mapt-KO+vehicle vs. Mapt$\mathrm{KO}+\mathrm{OTau} ; \mathrm{F}_{(1,15)}=45.32, P<0.0001$ in Mapt-KO+vehicle vs. Mapt-KO+ODQ; $\mathrm{F}_{(1,16)}=40.90, P<0.0001$ in Mapt-KO+vehicle vs. Mapt-KO+oA $3+0 \mathrm{ODQ} ; \mathrm{F}_{(1,15)}=$ 46.40, $P<0.0001$ in Mapt-KO+vehicle vs. Mapt-KO+OTau+ODQ; $n=8 \mathrm{Mapt-KO+vehicle,} n=10 \mathrm{Mapt}-\mathrm{KO}+\mathrm{OA} \beta, n=8 \mathrm{Mapt}-\mathrm{KO}+\mathrm{OTau}, n=9 \mathrm{Mapt}-\mathrm{KO}+\mathrm{ODQ}$, $n=10 \mathrm{Mapt}-\mathrm{KO}+\mathrm{OA} \beta+\mathrm{ODQ}, n=9 \mathrm{Mapt}-\mathrm{KO}+\mathrm{OTau}+\mathrm{ODQ}$ ). These experiments were interleaved with those displayed in B. (D) Analysis of slices displayed in B and C shows LTP impairment in WT slices treated with oA $\beta$ or oTau (Bonferroni's $P<0.0001$ vehicle vs. oA $\beta$ or oTau), but not in Mapt-KO slices treated with oA $\beta$ or oTau ( $P>0.05$ vehicle vs. oA $/ \mathrm{oTau})$ at 30 minutes after the tetanus. ODQ perfusion unraveled LTP defect in oA $\beta$ - or oTau-treated Mapt-KO slices $(P<0.05$ vehicle vs. ODQ+ OA $\beta /$ TTau $)$ at 30 minutes after the tetanus. ODQ did not further depress LTP in WT slices treated with oA $\beta$ or oTau $(P=1$ $\mathrm{ODQ}$ vs. OAB/OTau+ODQ) at 30 minutes after tetanus. (E) The same slices as in $\mathbf{D}$ showed LTP impairment at 120 minutes after the tetanus regardless of the treatment with oA /OTau/ODQ/ODQ+oA $/$ /oTau both in WT (Bonferroni's $P<0.0001)$ and Mapt-KO slices $(P<0.0001)$ at 120 minutes after tetanus. One-way ANOVA with Bonferroni correction (D and E). ${ }^{\S} P<0.0001$.

sion, similar to cultured hippocampal WT neurons, tau-KO neurons show a reduction of axonal transport after oA $\beta$ exposure, indicating that tau is not required for transport disruption (47), and likewise tau can impair axonal transport independent of $A \beta(48)$.

The early phase of LTP is protein synthesis independent whereas its late phase is protein synthesis dependent and requires gene transcription (49). Indeed, protein synthesis inhibitors do not prevent learning of tasks but disrupt memory of the training (50), supporting the view that there are different stages of memory with an early protein synthesis-independent stage and a late protein synthesis-dependent one that is required for consolidation of long-term memories (51). Based on this view and considering findings in the current manuscript, including protection against
$\mathrm{oA} \beta$ - and oTau-induced impairments by tau suppression of shortterm forms of plasticity and memory but not the long-term forms, we predict that although $\mathrm{AD}$ patients might respond to learning training after tau suppression, they may remain unable to consolidate memories.

In addition to our study's focus on lack of protection against damage of late phases of synaptic plasticity and long-term memory by tau suppression, we also interrogated the molecular basis of protection against the short-term plasticity defect by tau suppression. We found that inhibition of cGMP signaling via the sGC inhibitor ODQ blocks the neuroprotective effect of endogenous tau suppression against oA $\beta$ - and oTau-induced impairments of the early phase of synaptic plasticity. Moreover, the 


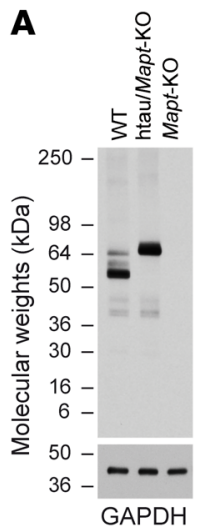

C

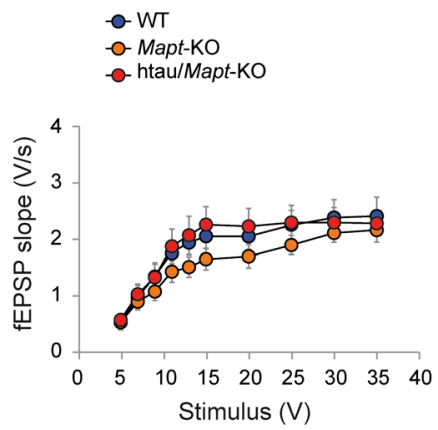

$\mathbf{F}$

o- WT, vehicle

- - WT, A $50 \mathrm{nM}$

- $\mathrm{O}$ Mapt-KO, vehicle

- - Mapt-KO, A $50 \mathrm{nM}$

-o- htau/Mapt-KO, vehicle

-o- htau/Mapt-KO, AB 50 nM

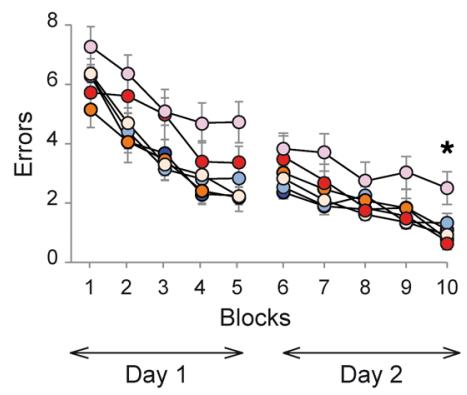

D $-W T$, vehicle

- $-\mathrm{WT}, \mathrm{A} \beta 50 \mathrm{nM}$

- Mapt-KO, vehicle

-O- Mapt-KO, A $50 \mathrm{nM}$

- htau/Mapt-KO, vehicle

-o- htau/Mapt-KO, A $50 \mathrm{nM}$

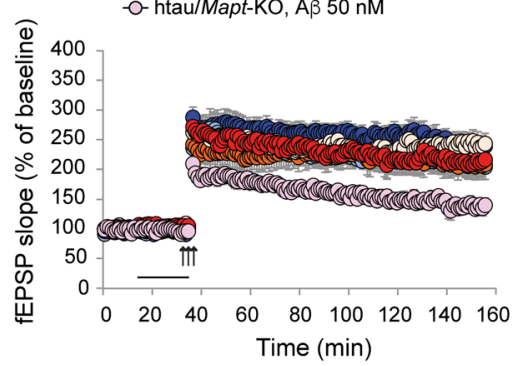

Total Tau sELISA

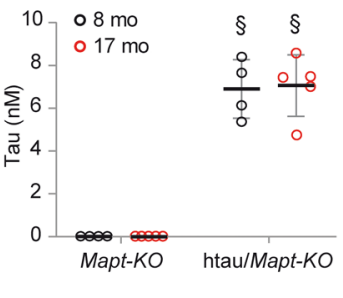

oTau sELISA

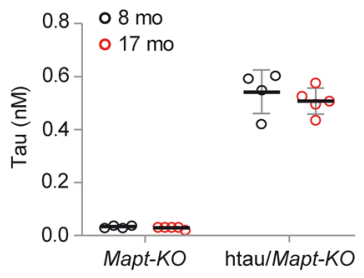

E

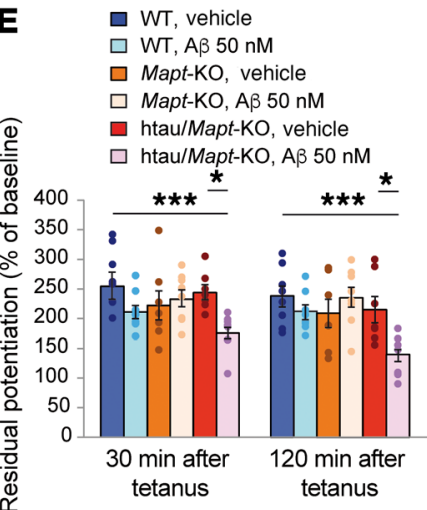

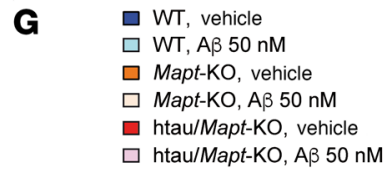

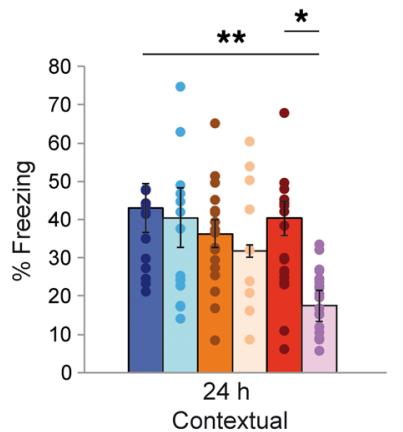

H

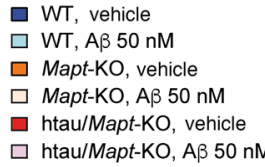
I $\square$ T, vehicle
$\square \mathrm{WT}, \mathrm{A} \beta 50 \mathrm{nM}$
$\square$ Mapt-KO, vehicle
$\square$ Mapt-KO, A $50 \mathrm{nM}$
$\square$ htau/Mapt-KO, vehicle
$\square$ htau/Mapt-KO, Aß $50 \mathrm{nM}$

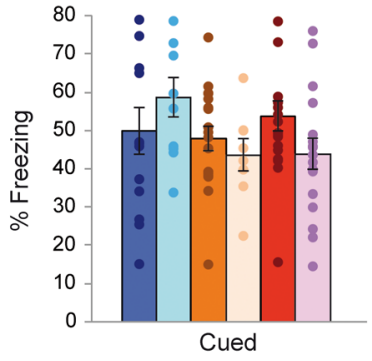

Figure 5. Reconstitution of Tau expression reestablishes oA $\beta$-induced disruption of short-term synaptic plasticity and memory. (A) Similar levels of human and murine tau expression in htau/Mapt-KO and WT mice. (B) Tau monomer (1.3-10 nM) standard curve $\left(r^{2}=0.92\right)$ used to interpolate total tau and tau aggregate $(0.16-1.3 \mathrm{nM})$ standard curve $\left(r^{2}=0.99\right)$ used to interpolate oTau. Assessment of total tau in hippocampus/cortex of 8-and 17-monthold htau/Mapt-KO mice (2-sample $t$ test, $t_{(6)}=17.983, P<0.0001$ compared with Mapt-KO at 8 months, $n=4 / 4 ; t_{(8)}=19.379$ compared with 17 months, $n=5 / 5$ ), and oTau levels ( 2 -sample $t$ test, $t(6)=12.044, P<0.0001$ compared with Mapt-KO at 8 months, $n=4 / 4 ; t(8)=21.354$ compared with 17 months, $n=5 / 5)$. Note the lack of signal in tau monomer standard curve in oTau assays demonstrating the specificity for oligomeric species. (C) Basal neurotransmission is similar in WT, Mapt-KO and htau/Mapt-KO slices (ANOVA for repeated measures $\mathrm{F}_{(2,40)}=3.865, P=0.639 ; n=15 / 11 / 17$, respectively). (D) Subtoxic extracellular oA $\beta\left(50 \mathrm{nM}\right.$ ) impairs LTP in htau/Mapt-KO slices (ANOVA for repeated measures $\mathrm{F}_{(1,15)}=33.474, P<0.0001 \mathrm{vs}$. WT+vehicle; $n=8 \mathrm{WT}+\mathrm{vehicle}$, $n=8 \mathrm{WT}+o \mathrm{~A} \beta, n=7 \mathrm{Mapt}-\mathrm{KO}+$ vehicle, $n=8 \mathrm{Mapt}-\mathrm{KO}+\mathrm{oA} \beta, n=7 \mathrm{htau} / \mathrm{Mapt}-\mathrm{KO}+$ vehicle, $n=9$ htau/Mapt-KO+oA $)$. (E) Analysis of slices displayed in D shows LTP impairment at 120 and 30 minutes after tetanus (1-way ANOVA with Bonferroni correction $P<0.05$ vs. WT or htau/Mapt-KO+vehicle). (F) Subtoxic oA $\beta$ (75 nM) impairs RAWM performance in htau/Mapt-KO mice (day 2 ANOVA for repeated measures $F_{(5,73)}=3.412, P=0.008 ; 1$-way ANOVA with Bonferroni correction $P<0.05$ vs. WT+vehicle; $P<0.005$ vs. htau/Mapt-KO+vehicle, block $10 ; n=13 \mathrm{WT}$ +vehicle, $n=13 \mathrm{WT}+0 \mathrm{~A} \beta, n=12 \mathrm{Mapt}$-KO+vehicle, $n=14 \mathrm{Mapt}-\mathrm{KO}+\mathrm{OA} \beta, n=14 \mathrm{htau} / \mathrm{Mapt}-\mathrm{KO}+$ vehicle, $n=14 \mathrm{htau} / \mathrm{Mapt}-\mathrm{KO}+\mathrm{oA} \beta)$. (G and $\mathbf{H}) \mathrm{oA} \beta 75 \mathrm{nM}$ impairs contextual fear memory in htau/Mapt-KO mice at 24 hours (1-way ANOVA with Bonferroni correction $\mathrm{F}_{(5,86)}=3.504, P<0.01$, Bonferroni's $P<0.05 ; n=13 \mathrm{WT}+$ vehicle, $n=16$ WT+oA $\beta, n=18$ Mapt-KO+vehicle, $n=9$ Mapt-KO+oA $\beta, n=19$ htau/Mapt-KO+vehicle, $n=18$ htau/Mapt-KO+oA $)$ and 30 minutes after training (1-way ANOVA with Bon-

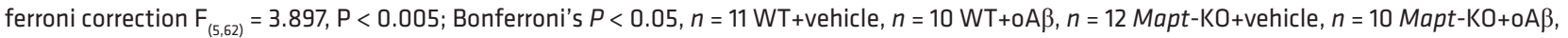
$n=12 \mathrm{htau} /$ Mapt-KO+vehicle, $n=13 \mathrm{htau} /$ Mapt $-\mathrm{KO}+\mathrm{OA} \beta)$. (I) Cued fear memory is similar in the 6 groups of mice displayed in $\mathbf{G}\left(1-\right.$ way ANOVA $F_{(5,79)}=$ $1.481, P=0.205) .{ }^{*} P<0.05 ;{ }^{*} P<0.01 ;{ }^{* *} P<0.005$. 
inhibitor did not further depress short-term plasticity in WT slices incubated with either oA $\beta$ or oTau, suggesting that oA $\beta$ and oTau impair plasticity via cGMP inhibition and not through additional independent mechanisms. These findings are consistent with the observation that the early phase of LTP requires an intact nitric oxide/cGMP signaling (37-39). Interestingly, both oA $\beta$ and oTau modulate hippocampal cGMP levels after LTP or memory induction $(36,40)$. Thus, it is likely that the protection against the oA $\beta$ - and oTau-induced defects of the early-phase of LTP in Mapt-KO mice is linked with cGMP signaling.

We found a reduction in basal synaptic transmission of TgAPP/Mapt-KO mice compared with the other groups, including the TgAPP mice. The analysis of neurotransmission in different transgenic models overexpressing mutated APP has often shown an impairment of basal neurotransmission at later stages than the LTP impairment $(52,53)$. Considering that tau performs multiple physiological functions (54), it is possible that tau suppression might exacerbate the negative effect of mutated APP overexpression onto basal neurotransmission. Independent of the impact on basal synaptic transmission, this raises an additional concern against the possibility of using tau suppression therapies. Given that the reduction of basal synaptic transmission was observed in animals overexpressing mutated APP in a complete absence of tau, one cannot conclude that a partial tau suppression such as the one obtained with tau antisense oligonucleotides or antibodies would definitively impair synaptic function in $\mathrm{AD}$ patients.

Similar to long-term contextual fear memory, we did not find a rescue of the cued fear memory impairment following tau suppression in TgAPP/Mapt-KO mice. Cued fear conditioning is an amygdala-dependent and hippocampus-independent task (55). Interestingly, the amygdala is affected both in $\mathrm{AD}$ mouse models and $\mathrm{AD}$ patients (56). It characteristically shows shrinkage, distortion and loss of neurons, and widespread gliosis in AD patients (57-59). Moreover, emotional memory impairment in AD patients positively correlates with amygdala atrophy (56). Altogether, these findings suggest that tau suppression will not rescue the defect in emotional memory of $\mathrm{AD}$ patients.

Consistent with the findings on LTP and memory, analysis of amyloid load did not show any difference between TgAPP/ Mapt-KO and TgAPP mice. These results were similar to studies in J20 mice crossed with Mapt-KO animals (1), supporting the observation that $A \beta$-induced synaptic plasticity and memory loss are independent of tau suppression. Most importantly, they extend to $\mathrm{AD}$ histopathology the concept that tau suppression is not beneficial against $\mathrm{AD}$ progression.

Another finding in our studies is that exogenously applied oTau impairs the late phase of LTP and long-term memory regardless of endogenous tau suppression. This is interesting because it suggests that oTau behaves similar to oA $\beta$. Consistent with this conclusion, the 2 proteins share several biochemical, physiological, and pathological features in common (26). Both are involved in synaptic plasticity in the normal healthy brain $(60,61)$, whereas in the diseased brain they form toxic oligomeric species, probably because they form $\beta$-sheets $(10,62-64)$. Moreover, subtoxic doses of oTau and oA $\beta$ produce coordinated changes in synaptic plasticity and memory (10). Most importantly, this finding highlights differ- ences in the mechanism of action of tau in its native form versus oligomers derived from it.

We found that oTau does not impair the initial phase of LTP and short-term memory in Mapt-KO mice. This finding suggests that oTau needs endogenous tau to affect the initial phase of LTP and short-term memory. Moreover, it shows an additional parallelism between tau and $A \beta$, in that tau suppression protects against the early damage of synaptic plasticity and memory caused by both oA $\beta$ and oTau.

Overexpression of human WT tau abolishes the protective effect tau suppression has against $A \beta$-induced impairment of the initial phase of LTP and short-term memory. These experiments confirm that tau is genuinely needed for these impairments to occur. Additionally, they are consistent with the idea that different memory types exist: a tau-dependent one and a tauindependentone. Tothisend, themolecularmechanisms of thetauindependent memory could involve APP, as binding between $\mathrm{APP}$ and tau or $\mathrm{A} \beta$ is required for the detrimental effects of $\mathrm{A} \beta$ and tau on long-term synaptic plasticity and memory (19, 27-31). Additionally, fragments of APP processing are gaining increased attention. They could be modulated differentially in response to the various single and combined alterations of $A \beta$ and tau. In particular, APP- $\beta \mathrm{CTF}$ is emerging as a highly relevant pathogenic factor in $\mathrm{AD}$ and previous work has shown similarities in the profile of synaptic and cognitive effects induced by altered levels or distributions of APP- $\beta$ CTF (65-70).

The impairment of LTP and memory was present with subtoxic doses of oA $\beta$ in htau/Mapt-KO mice. This is probably dependent on the fact that these mice produce low amounts of oTau, which causes full-blown impairments when combined with low doses of oA $\beta$. Consistent with this finding, oA $\beta$ and oTau act in cooperation when they determine LTP and memory impairment (10). Moreover, dose is not the only variable in our experimental paradigms that might have affected outcome. Other important variables that one should take into account when interpreting results are the age of the animals and the duration of the treatment. For instance, we found memory defects in Mapt-KO mice after the age of 10 months. For this reason, we chose to perform experiments at an age in which tau suppression does not interfere with the interpretation of our findings. Additionally, we crossed Mapt-KO animals with TgAPP mice with chronic expression and accumulation of naturally produced $A \beta$, to extend the validity of findings from experiments with acute exposure to oA $\beta$.

A straightforward conclusion from our experiments is that anti-A $\beta$ and anti-tau therapies alone are unlikely to effectively treat all AD symptoms. Thus, tau-targeting therapies or early intervention against $\mathrm{A} \beta$ are unlikely the solution to treat $\mathrm{AD}$, and these data call for a reassessment of many clinical trials based on the amyloid hypothesis. Most importantly, our findings suggest that therapies that simultaneously target $A \beta$ and tau might effectively improve LTP and memory. This might be achieved by either combining anti$\mathrm{A} \beta$ and anti-tau therapeutics, or more likely, given that the physiological functions of these proteins might render these therapeutics not clinically viable $(71,72)$, targeting substrates downstream of both peptides through either personalized medicine approaches or drugs acting on second messenger systems shared by the 2 proteins and relevant to synaptic plasticity and memory. 


\section{Methods}

Animals. The following groups of mice were used: (a) Mapt-KO and WT littermates (32, 41) (https://www.jax.org/strain/007251); (b) transgenic mice overexpressing human APP carrying the Swedish (APP KM670/671NL) and the Indiana (V717F) mutations named TgAPP mice (33) with their TgAPP/Mapt-KO and Mapt-KO littermates obtained by crossing TgAPP in a tau-hemizygous background; (c) htau/Mapt-KO mice obtained by crossing htau mice in a murine tau-hemyzygous background to generate htau/Mapt-KO mice and siblings (41). The htau animals express WT, full-length human tau $(2 \mathrm{~N} 4 \mathrm{R})$ driven by the prion promoter and were generated using the same approach as previously described for the R406W and P301L mutant transgenes $(73,74)$. Mice were obtained from breeding colonies kept in the animal facility of the University of Toronto. PCR on tail samples was used for genotyping, as previously described (73). Animals were maintained on a 12-hour light/12-hour dark cycle, in a temperature- and humidity-controlled room. Food and water were available ad libitum. Mice were allocated to a specific treatment and paradigm by a randomization procedure. Investigators who performed the experiments were blind in respect to genotype and treatment. All experiments were performed on sex-balanced groups. Mice were used at 4 to 6 months of age, unless otherwise stated in Results.

Electrophysiology. Electrophysiological experiments were performed as previously described (36). Briefly, following their cutting, transverse hippocampal slices $(400 \mu \mathrm{m})$ were transferred to a recording chamber where they were maintained at $29^{\circ} \mathrm{C}$ and perfused with ACSF (flow rate $2 \mathrm{~mL} / \mathrm{min}$; continuously bubbled with $95 \% \mathrm{O}_{2}$ and $5 \% \mathrm{CO}_{2}$ ), consisting of $\mathrm{NaCl}(124.0 \mathrm{mM}), \mathrm{KCl}(4.4 \mathrm{mM}), \mathrm{Na}_{2} \mathrm{HPO}_{4}(1.0 \mathrm{mM})$, $\mathrm{NaHCO}_{3}(25.0 \mathrm{mM}), \mathrm{CaCl}_{2}(2.0 \mathrm{mM}), \mathrm{MgCl}_{2}(2.0 \mathrm{mM})$, and glucose (10.0 $\mathrm{mM})$. Stimulation of the Schaeffer collateral fibers through a bipolar tungsten electrode permitted the recording of field extracellular recordings (fEPSP) in CA1 stratum radiatum with a glass pipette filled with ACSF. After evaluation of input-output relationship to measure basal synaptic transmission, a 15-minute baseline was recorded every minute at an intensity eliciting a response approximately $35 \%$ of the maximum evoked response. $\mathrm{A} \beta$ and tau were applied for 20 minutes after recording of the baseline. For experiments with no application of $\mathrm{A} \beta$ and tau, the baseline was recorded for 20 minutes before eliciting potentiation. Additionally, ODQ (Cayman Chemical Company) was applied for 10 minutes before tetanus in a few experiments. LTP was induced through a theta-burst stimulation (4 pulses at $100 \mathrm{~Hz}$, with the bursts repeated at $5 \mathrm{~Hz}$ and 3 tetani of 10-burst trains administered at 15-second intervals). Responses were recorded for 2 hours after tetanization and measured as fEPSP slope expressed as percentage of baseline.

Behavior. Intrahippocampal infusions of oA $\beta$ and oTau were performed following stereotaxic surgery for cannula implantation, as previously described (19). Briefly, while anesthetized with Avertin (500 $\mathrm{mg} / \mathrm{kg}$ ), mice were implanted with a 26-gauge guide cannula in the dorsal part of the hippocampi (coordinates from bregma: posterior $2.46 \mathrm{~mm}$, lateral $1.50 \mathrm{~mm}$ to a depth of $1.30 \mathrm{~mm}$ ). After 6 to 8 days of recovery, mice were bilaterally infused with oA $\beta$ or oTau or vehicle (final volume of $1 \mu \mathrm{L}$ over 60 seconds). During infusion, animals were handled gently to minimize stress. In some animals, a solution of $4 \%$ methylene blue was infused for localization of infusion cannulas after behavioral studies.

The RAWM test was performed over 2 days as previously described (75). During the first day, mice were trained in 15 trials to identify the platform location in a goal arm by alternating between a visible and a hidden platform from trial 1 to 12 . In the last 4 trials only a hidden platform was used. During the second day, the platform was hidden throughout trial 1 to 15 . Errors were counted when the mice entered an arm with no platform, or failed to select an arm for 15 seconds and the mouse was gently pulled back to the start arm. Each trial lasted up to 1 minute. At the end of each trial, mice rested on the platform for 15 seconds. The goal arm was maintained constant for all trials, with a different starting arm on successive trials. Data were analyzed and displayed as averages of blocks of 3 trials. Following RAWM testing, mice underwent a visible platform test to control for possible motivational, visual, and motor defects. This consisted of a 2-day test, with 2 sessions per day (each consisting of three 1-minute trials) in which the time taken to reach a visible platform (randomly positioned in a different place each time) marked with a green flag was recorded.

Fear conditioning was performed as previously described $(75,76)$. Briefly, mice were handled once a day for 3 days before behavioral experiments. During the first day, mice were placed in the conditioning chamber for 2 minutes before the onset of a discrete tone (conditioned stimulus [CS], a sound that lasted 30 seconds at $2800 \mathrm{~Hz}$ and $85 \mathrm{~dB}$ ). In the last 2 seconds of the CS, mice were given a foot shock (unconditioned stimulus [US]) of $0.80 \mathrm{~mA}$ for 2 seconds through the bars of the floor. After the CS/US pairing, the mice were left in the conditioning chamber for 30 seconds and then they were placed back in their home cages. Freezing behavior (defined as the absence of all movement except for that necessitated by breathing) was measured. The contextual fear learning was evaluated during the second day for 5 consecutive minutes. The cued fear learning was evaluated during the third day by placing the mouse in a novel context for 2 minutes (pre-CS test), after which they were exposed to the CS for 3 minutes (CS test). Sensory perception of the shock (determined 24 hours after the cued test through threshold assessment) started with a foot shock of $0.1 \mathrm{~mA}$ that increased by $0.1 \mathrm{~mA}$ every 30 seconds. We recorded the first visible, motor, and vocal response.

Open field was performed as previously described (10). Briefly, mice were left in a white arena divided into sectors (periphery and center) by black lines. Each mouse was permitted to freely explore the arena for 5 minutes on 2 consecutive days. We scored the percentage of time spent in the center and the number of entries into the center.

Preparation of $A \beta$ and tau oligomers. Human $A \beta_{42}$ oligomerization was obtained as previously described (36). Briefly, a protein film was prepared by dissolving $\mathrm{A} \beta_{42}$ lyophilized powder (Biopolymer Laboratory, UCLA) in 1,1,1,3,3,3-Hexafluoro-2-Propanol (HFIP) and subsequent incubation for 2 hours at room temperature to allow complete monomerization. The $\mathrm{A} \beta$ film was dissolved in dimethylsulfoxide (DMSO), sonicated for 15 minutes, aliquoted, and stored at $-20^{\circ} \mathrm{C}$. To oligomerize the peptide, phosphate buffered saline (PBS) was added to an aliquot of DMSO-A $\beta$ to obtain a $5 \mathrm{mM}$ solution that was incubated for 12 hours at $4^{\circ} \mathrm{C}$. This oligomerized $A \beta$ solution was then diluted to the final concentration in artificial cerebrospinal fluid (ACSF) consisting of $124.0 \mathrm{mM} \mathrm{NaCl}, 4.4 \mathrm{mM} \mathrm{KCl}, 1.0 \mathrm{mM} \mathrm{Na}_{2} \mathrm{HPO}_{4}, 25.0 \mathrm{mM}$ $\mathrm{NaHCO}_{3}, 2.0 \mathrm{mM} \mathrm{CaCl}$, and $2.0 \mathrm{mM} \mathrm{MgCl}_{2}$. The $\mathrm{A} \beta$ preparation was monitored through Western blot in which $\mathrm{A} \beta$ samples (prepared in nondenaturing/nonreducing conditions before loading) were resolved by a denaturing Tris-Tricine SDS-PAGE and probed with antihuman A $\beta$ monoclonal antibody 6E10 (BioLegend; catalog SIG-39320; dilution 1:1000). 
Human recombinant tau $4 \mathrm{R} / 2 \mathrm{~N}$ was used to obtain tau as previously described $(10,19,77)$. Oligomerization was achieved through introduction of disulfide bonds via incubation with $1 \mathrm{mM} \mathrm{H}_{2} \mathrm{O}_{2}$ at room temperature for 20 hours, followed by centrifugation in PES at $4000 \mathrm{~g}$. The resulting material was used for the experiments. The tau preparation was monitored through Western blot without reducing agent, as described (77). The samples were loaded to $10 \%$ Tris-Acetate gels that transferred on nitrocellulose membrane, following a common Western blot protocol (anti-tau antibody RabMad EP2456Y; catalog ab76128; dilution 1:1000).

Immunohistochemistry. For immunohistochemistry and amyloid load analyses, brain hemispheres were fixed in 10\% formalin (MilliporeSigma) overnight at $4^{\circ} \mathrm{C}$ then immersed in $70 \%$ ethanol. Serial sections $(5 \mu \mathrm{m})$ of paraffin-embedded tissue were stained for amyloid plaques using an A $\beta$-specific antibody (4G8, BioLegend; catalog 800701; dilution 1:200) or tau using the TAU-5 antibody (Thermo Fisher Scientific; catalog MA5-12808; dilution 1:500). Plaque densities for the different groups of transgenic mice were determined as previously described (78). Briefly, immunostained sections $(5 \mu \mathrm{m})$ were scanned with Mirax Scan (Zeiss) and assessed using ImageScope (Aperio). Slides were scanned using the Mirax Scan v. 1.11 software and Zeiss Mirax Slide Scanner at $\times 20$ magnification with a Zeiss $\times 20 / 0.8$ objective lens and a Marlin F146-C CCD camera. The rendered digital images were analyzed using the Color Deconvolution Algorithm in the Aperio Imagescope software, as previously described (79). For tau immunostaining, Cy3-conjugated anti-mouse (Jackson ImmunoResearch; catalog 115-165-146; dilution 1:1000) was used as secondary antibody and cellular nuclei were labeled with DAPI.

Western blotting. Brain tissues were homogenized in RIPA buffer and separated on a 4\%-20\% Tris-Glycine gradient gel (10 $\mu$ g total protein/lane) and probed with a rabbit polyclonal antibody to human tau (Agilent/DAKO; catalog A002401-2; dilution of 1:100,000). Equal amounts of proteins were loaded into each lane. To confirm equal loading, blots were reprobed with corresponding antibody for GADPH (Origene; catalog TA8025198BM; dilution of 1:2000).

Total and oTau sandwich enzyme-linked immunosorbent assay (sELISA). Brain tissue from the combined hippocampus and cortex was homogenized in lysis buffer (20 mM Tris; pH 7.4, 0.25M sucrose, 1 mM EDTA, 1 mM EGTA, supplemented with protease inhibitors and PhosStop from MilliporeSigma) using sonication. Then, 0.1\% Triton $\mathrm{X}-100$ was added to the samples and lysates were spun at $12,000 \mathrm{~g}$ for 20 minutes at $4{ }^{\circ} \mathrm{C}$ to remove cellular debris. The supernatant was collected and a protein assay was used to determine total protein content. Samples were assayed in total tau and oligomeric tau sELISAs using methods similar to those previously described (80-82). Recombinant tau proteins were generated as described previously (83). Monomeric tau- and arachidonic acid-induced aggregates were produced as described (83). For total tau assays, the capture antibody was Tau12 (aa8-21, catalog AB_2721192) $(80,84)$ and $100 \mu \mathrm{g}$ lysate protein was used. For oTau assays, the oligomer-specific, TOC1 monoclonal antibody (aa209-225, catalog AB_2832939) $(85,86)$, was used for capture and $25 \mu$ g lysate protein was used. The polyclonal pan-tau antibody, R1 (catalog AB_2832929) (87), was used for detection of captured tau. Monomeric tau- and arachidonic acid-induced aggregates were produced as described (83). Recombinant protein standards consisting of monomeric tau (10-1.3 nM) or aggregated tau (0.16-1.3 nM) were used to estimate the level of tau present in total tau assays or oTau assays, respectively. Absorbance was read at $450 \mathrm{~nm}$ using a spectrophotometer. Absorbance data from the standard curves were used to convert the brain lysate sample data to tau levels.

Statistics. Investigators who performed the experiments were blinded with respect to treatment and genotype. Pairing between raw data and the corresponding group was performed at the end of each experimental setting. Preestablished inclusion criteria were used to select hippocampal slices for electrophysiological recordings (healthy slices with smooth edges and surface) and mice for behavioral studies (animals in general good health, averaged weight $28 \pm 2 \mathrm{~g}$ for females and $30 \pm 3 \mathrm{~g}$ for males). Animals were allocated to a specific group by a randomization procedure. Sample size was calculated by G-Power 3.1 software. Power analyses $(\alpha=0.05$, power $1-\beta=0.80)$ suggested a minimum of 6 slices (electrophysiology) and 8 mice (behavioral studies) to obtain an effect size equal to 0.62 .

After data collection, statistical analysis was performed using Systat 9 software. A preliminary analysis of normal distribution was performed by Shapiro-Wilk normality test. For electrophysiological recordings, 1 to 2 slices were recorded from the same mouse and the reported $N$ corresponds to the number of slices. Results were analyzed in pClamp 10 (Molecular Devices) and compared by ANOVA with repeated measures for input/output relationship and LTP curves, 1-way ANOVA with Bonferroni's post hoc corrections for 26th-30th and the 116th-120th recording points after tetanus. For behavioral experiments, mice were distributed in a balanced fashion with respect to sex and genotype and for each condition mice were trained and tested in 3 to 4 separate sets of experiments. Errors in the RAWM were manually counted. Freezing, latency, time spent in the center of the arena and number of entries into the center were scored by using a video-tracking recording system. We used 1-way ANOVA with Bonferroni's post hoc correction or ANOVA with repeated measures for comparisons among the groups of mice. Two-sample $t$ test was used when comparing 2 conditions. Data were expressed as mean \pm SEM. The level of significance was set at $P$ less than 0.05 .

Study approval. All protocols involving animals complied with the Animal Research: Reporting of In Vivo Experiments (ARRIVE) guidelines and were approved by the Institutional Animal Care and Use Committee at Columbia University, the University of Toronto, and the University of Catania.

\section{Author contributions}

DP contributed to project conceptualization, formal analysis, and investigation, and to the writing of the original draft, review, editing, and visualization of the manuscript. EKA contributed to the electrophysiological experiments and the writing of the manuscript. AS performed the behavioral experiments. HZ performed electrophysiological experiments. EC, EZ, EA, and DDLP performed biochemical experiments. MF contributed to the electrophysiological experiments and conceptualization of the manuscript. CG contributed to project conceptualization, and to the writing, review, and editing of the manuscript. LD contributed to project conceptualization, provided resources, and participated in the writing, review, and editing of the manuscript. NMK and PEF contributed to project conceptualization and formal analysis of the data, provided resources, participated in the writing, review, and editing of the manuscript, and helped with funding acquisition. OA contributed to project conceptualization, wrote the original 
draft and its revision, supervised the whole work, administered the project, and acquired funds.

\section{Acknowledgments}

This work was supported by NIH grants R01AG049402 (to OA), R01NS082730, and R01AG044372 (to NMK) and by Canadian Institutes of Health Research grant MOP-115056 (to PEF). We thank Rosemary Ahrens for maintaining the mouse colonies and Kyung Han for assistance with the biochemical studies.

Address correspondence to: Ottavio Arancio, P\&S \#12-420D, Columbia University Irving Medical Center, 630 West 168th Street, New York, New York, USA. 10032. Phone: 212.342.0533; Email: oa1@columbia.edu.
1. Roberson ED, et al. Reducing endogenous tau ameliorates amyloid beta-induced deficits in an Alzheimer's disease mouse model. Science. 2007;316(5825):750-754.

2 . Vossel KA, et al. Tau reduction prevents A $\beta$-induced axonal transport deficits by blocking activation of GSK3 $\beta$. JCell Biol. 2015;209(3):419-433.

3. Vossel KA, et al. Tau reduction prevents Abetainduced defects in axonal transport. Science. 2010;330(6001):198.

4. Miyamoto T, et al. Phosphorylation of tau at Y18, but not tau-fyn binding, is required for tau to modulate NMDA receptor-dependent excitotoxicity in primary neuronal culture. Mol Neurodegener. 2017;12(1):41.

5. Shipton OA, et al. Tau protein is required for amyloid \{beta\}-induced impairment of hippocampal long-term potentiation. J Neurosci. 2011;31(5):1688-1692.

6. Fuster-Matanzo A, Hernández F, Ávila J. Tau spreading mechanisms; implications for dysfunctional tauopathies. Int J Mol Sci. 2018;19(3):E645.

7. Vargas-Caballero M, et al. Wild-type, but not mutant $\mathrm{N} 296 \mathrm{H}$, human tau restores A $\beta$-mediated inhibition of LTP in Tau ${ }^{-/-}$mice. Front Neurosci. 2017;11:201.

8. Brody DL, et al. Amyloid-beta dynamics correlate with neurological status in the injured human brain. Science. 2008;321(5893):1221-1224.

9. Cirrito JR, et al. Synaptic activity regulates interstitial fluid amyloid-beta levels in vivo. Neuron. 2005;48(6):913-922.

10. Fá M, et al. Extracellular Tau oligomers produce an immediate impairment of LTP and memory. Sci Rep. 2016;6:19393.

11. Kamenetz F, et al. APP processing and synaptic function. Neuron. 2003;37(6):925-937.

12. Pooler AM, Phillips EC, Lau DH, Noble W, Hanger DP. Physiological release of endogenous tau is stimulated by neuronal activity. EMBO Rep. 2013;14(4):389-394.

13. Yamada K, et al. Neuronal activity regulates extracellular tau in vivo. J Exp Med. 2014;211(3):387-393.

14. Frost B, Jacks RL, Diamond MI. Propagation of tau misfolding from the outside to the inside of a cell. J Biol Chem. 2009;284(19):12845-12852.

15. Holmes BB, et al. Heparan sulfate proteoglycans mediate internalization and propagation of specific proteopathic seeds. Proc Natl Acad Sci USA. 2013;110(33):E3138-E3147.

16. Lai AY, McLaurin J. Mechanisms of amyloid-beta peptide uptake by neurons: the role of lipid rafts and lipid raft-associated proteins. Int J Alzheimers Dis. 2010;2011:548380.

17. Luo W, Liu W, Hu X, Hanna M, Caravaca A, Paul SM. Microglial internalization and degradation of pathological tau is enhanced by an anti-tau monoclonal antibody. Sci Rep. 2015;5:11161.

18. Piacentini R, et al. Reduced gliotransmitter release from astrocytes mediates tau-induced synaptic dysfunction in cultured hippocampal neurons. Glia. 2017;65(8):1302-1316.

19. Puzzo D, et al. LTP and memory impairment caused by extracellular $A \beta$ and tau oligomers is APP-dependent. Elife. 2017;6:e26991.

20. Wu JW, et al. Small misfolded tau species are internalized via bulk endocytosis and anterogradely and retrogradely transported in neurons. J Biol Chem. 2013;288(3):1856-1870.

21. Lv ZY, Tan CC, Yu JT, Tan L. Spreading of pathology in Alzheimer's disease. Neurotox Res. 2017;32(4):707-722.

22. Lasagna-Reeves CA, et al. Identification of oligomers at early stages of tau aggregation in Alzheimer's disease. FASEB J. 2012;26(5):1946-1959.

23. Selkoe DJ. Soluble oligomers of the amyloid beta-protein impair synaptic plasticity and behavior. Behav Brain Res. 2008;192(1):106-113.

24. Ondrejcak T, et al. Cellular prion protein mediates the disruption of hippocampal synaptic plasticity by soluble tau in vivo. J Neurosci. 2018;38(50):10595-10606.

25. Pickett EK, et al. Amyloid beta and tau cooperate to cause reversible behavioral and transcriptional deficits in a model of Alzheimer's disease. Cell Rep. 2019;29(11):3592-3604.e5.

26. Gulisano W, et al. Role of amyloid- $\beta$ and tau proteins in Alzheimer's disease: confuting the amyloid cascade. JAlzheimers Dis. 2018;64(s1):S611-S631.

27. Fogel H, et al. APP homodimers transduce an amyloid- $\beta$-mediated increase in release probability at excitatory synapses. Cell Rep. 2014;7(5):1560-1576.

28. Lorenzo A, et al. Amyloid beta interacts with the amyloid precursor protein: a potential toxic mechanism in Alzheimer's disease. Nat Neurosci. 2000;3(5):460-464.

29. Shaked GM, Kummer MP, Lu DC, Galvan V, Bredesen DE, Koo EH. Abeta induces cell death by direct interaction with its cognate extracellular domain on APP (APP 597-624). FASEB J. 2006;20(8):1254-1256.

30. Takahashi M, et al. Extracellular association of APP and tau fibrils induces intracellular aggregate formation of tau. Acta Neuropathol. 2015;129(6):895-907.

31. Van Nostrand WE, et al. Localization of a fibrillar amyloid beta-protein binding domain on its precursor. J Biol Chem. 2002;277(39):36392-36398.

32. Dawson HN, Ferreira A, Eyster MV, Ghoshal N, Binder LI, Vitek MP. Inhibition of neuronal maturation in primary hippocampal neurons from tau deficient mice. JCell Sci. 2001;114(Pt 6):1179-1187. 33. Chishti MA, et al. Early-onset amyloid depo- sition and cognitive deficits in transgenic mice expressing a double mutant form of amyloid precursor protein 695. J Biol Chem. 2001;276(24):21562-21570.

34. Knock E, et al. SUMO1 impact on Alzheimer disease pathology in an amyloid-depositing mouse model. Neurobiol Dis. 2018;110:154-165.

35. Steele JW, et al. Early fear memory defects are associated with altered synaptic plasticity and molecular architecture in the TgCRND8 Alzheimer's disease mouse model. JComp Neurol. 2014;522(10):2319-2335.

36. Puzzo D, Vitolo O, Trinchese F, Jacob JP, Palmeri A, Arancio O. Amyloid-beta peptide inhibits activation of the nitric oxide/cGMP/cAMPresponsive element-binding protein pathway during hippocampal synaptic plasticity. J Neurosci. 2005;25(29):6887-6897.

37. Hawkins RD, Son H, Arancio O. Nitric oxide as a retrograde messenger during long-term potentiation in hippocampus. Prog Brain Res. 1998;118:155-172.

38. Son H, Lu YF, Zhuo M, Arancio O, Kandel ER, Hawkins RD. The specific role of cGMP in hippocampal LTP. Learn Mem. 1998;5(3):231-245.

39. Boulton CL, Southam E, Garthwaite J. Nitric oxide-dependent long-term potentiation is blocked by a specific inhibitor of soluble guanylyl cyclase. Neuroscience. 1995;69(3):699-703.

40. Acquarone E, et al. Synaptic and memory dysfunction induced by tau oligomers is rescued by up-regulation of the nitric oxide cascade. $\mathrm{Mol}$ Neurodegener. 2019;14(1):26.

41. Wijesekara N, Gonçalves RA, Ahrens R, De Felice FG, Fraser PE. Tau ablation in mice leads to pancreatic $\beta$ cell dysfunction and glucose intolerance. FASEB J. 2018;32(6):3166-3173.

42. Nicholls RE, et al. PP2A methylation controls sensitivity and resistance to $\beta$-amyloid-induced cognitive and electrophysiological impairments. Proc Natl Acad Sci USA. 2016;113(12):3347-3352.

43. Puzzo D, et al. Picomolar amyloid-beta positively modulates synaptic plasticity and memory in hippocampus. J Neurosci. 2008;28(53):14537-14545.

44. Morris M, Maeda S, Vossel K, Mucke L. The many faces of tau. Neuron. 2011;70(3):410-426.

45. Ittner LM, et al. Dendritic function of tau mediates amyloid-beta toxicity in Alzheimer's disease mouse models. Cell. 2010;142(3):387-397.

46. Roberson ED, et al. Amyloid- $\beta /$ Fyn-induced synaptic, network, and cognitive impairments depend on tau levels in multiple mouse models of Alzheimer's disease. JNeurosci. 2011;31(2):700-711.

47. Ramser EM, et al. Amyloid- $\beta$ oligomers induce tau-independent disruption of BDNF axonal transport via calcineurin activation in cultured hippocampal neurons. Mol Biol Cell. 2013;24(16):2494-2505. 
48. Combs B, Mueller RL, Morfini G, Brady ST, Kanaan NM. Tau and axonal transport misregulation in tauopathies. Adv Exp Med Biol. 2019;1184:81-95.

49. Huang EP. Synaptic plasticity: going through phases with LTP. Curr Biol. 1998;8(10):R350-R352.

50. Agranoff BW, Davis RE, Brink JJ. Chemical studies on memory fixation in goldfish. Brain Res. 1966;1(3):303-309.

51. McGaugh JL. Memory--a century of consolidation. Science. 2000;287(5451):248-251.

52. Larson J, Lynch G, Games D, Seubert P. Alterations in synaptic transmission and long-term potentiation in hippocampal slices from young and aged PDAPP mice. Brain Res. 1999;840(1-2):23-35.

53. Trinchese F, Liu S, Battaglia F, Walter S, Mathews $\mathrm{PM}$, Arancio O. Progressive age-related development of Alzheimer-like pathology in APP/PS1 mice. Ann Neurol. 2004;55(6):801-814.

54. Guo T, Noble W, Hanger DP. Roles of tau protein in health and disease. Acta Neuropathol. 2017;133(5):665-704.

55. Phillips RG, LeDoux JE. Differential contribution of amygdala and hippocampus to cued and contextual fear conditioning. Behav Neurosci. 1992;106(2):274-285.

56. Knafo S. Amygdala in Alzheimer's disease. In: Ferry B, ed. The Amygdala - A Discrete Multitasking Manager. IntechOpen; 2012:375-383.

57. Cuénod CA, et al. Amygdala atrophy in Alzheimer's disease. An in vivo magnetic resonance imaging study. Arch Neurol. 1993;50(9):941-945.

58. Herzog AG, Kemper TL. Amygdaloid changes in aging and dementia. Arch Neurol. 1980;37(10):625-9.

59. Vereecken TH, Vogels OJ, Nieuwenhuys R. Neuron loss and shrinkage in the amygdala in Alzheimer's disease. Neurobiol Aging. 1994;15(1):45-54.

60. Biundo F, Del Prete D, Zhang H, Arancio O, D'Adamio L. A role for tau in learning, memory and synaptic plasticity. Sci Rep. 2018;8(1):3184.

61. Puzzo D, Arancio O. Amyloid- $\beta$ peptide: Dr. Jekyll or Mr. Hyde? J Alzheimers Dis. 2013;33 Suppl 1:S111-S120.

62. Gendreau KL, Hall GF. Tangles, toxicity, and tau secretion in $\mathrm{AD}$ - new approaches to a vexing problem. Front Neurol. 2013;4:160.

63. Hayden EY, Teplow DB. Amyloid $\beta$-protein oligomers and Alzheimer's disease. Alzheimers Res Ther. 2013;5(6):60.

64. Lasagna-Reeves CA, Castillo-Carranza DL, Sengupta U, Clos AL, Jackson GR, Kayed R. Tau oligomers impair memory and induce synaptic and mitochondrial dysfunction in wild-type mice. Mol Neurodegener. 2011;6:39.

65. Kim S, et al. Evidence that the rab5 effector APPL mediates APP- $\beta$ CTF-induced dysfunction of endosomes in Down syndrome and Alzheimer's disease. Mol Psychiatry. 2016;21(5):707-716.

66. Tamayev R, Matsuda S, Arancio O, D’Adamio L. $\beta$-but not $\gamma$-secretase proteolysis of APP causes synaptic and memory deficits in a mouse model of dementia. EMBO Mol Med. 2012;4(3):171-179.

67. Kwart D, et al. A large panel of isogenic APP and PSEN1 mutant human iPSC neurons reveals shared endosomal abnormalities mediated by APP $\beta$-CTFs, not A $\beta$. Neuron. 2019;104(2):256-270.e5.

68. Tamayev R, D'Adamio L. Inhibition of $\gamma$-secretase worsens memory deficits in a genetically congruous mouse model of Danish dementia. $\mathrm{Mol}$ Neurodegener. 2012;7:19.

69. Jiang Y, et al. Lysosomal dysfunction in Down syndrome is APP-dependent and mediated by APPBCTF (C99). J Neurosci. 2019;39(27):5255-5268.

70. Jiang Y, et al. Partial BACE1 reduction in a Down syndrome mouse model blocks Alzheimer-related endosomal anomalies and cholinergic neurodegeneration: role of APP-CTF. Neurobiol Aging. 2016;39:90-98.

71. Puzzo D, et al. Endogenous amyloid- $\beta$ is necessary for hippocampal synaptic plasticity and memory. Ann Neurol. 2011;69(5):819-830.

72. Wang Y, Mandelkow E. Tau in physiology and pathology. Nat Rev Neurosci. 2016;17(1):5-21.

73. Ikeda $\mathrm{M}$, et al. Accumulation of filamentous tau in the cerebral cortex of human tau R406W transgenic mice. Am J Pathol. 2005;166(2):521-531.

74. Murakami T, et al. Cortical neuronal and glial pathology in TgTauP301L transgenic mice: neuronal degeneration, memory disturbance, and phenotypic variation. Am J Pathol. 2006;169(4):1365-1375

75. Watterson DM, et al. Development of novel in vivo chemical probes to address CNS protein kinase involvement in synaptic dysfunction. PLOS ONE. 2013;8(6):e66226.

76. Fiorito J, et al. Synthesis of quinoline derivatives: discovery of a potent and selective phosphodiesterase 5 inhibitor for the treatment of Alzheimer's disease. Eur JMed Chem. 2013;60:285-294.

77. Argyrousi EK, Staniszewski A, Nicholls RE, Arancio O. Preparation of tau oligomers after the protein extraction from bacteria and brain cortices. Methods Mol Biol. 2018;1779:85-97.

78. Satoh K, Abe-Dohmae S, Yokoyama S, St George-Hyslop P, Fraser PE. ATP-binding cassette transporter A7 (ABCA7) loss of function alters Alzheimer amyloid processing. J Biol Chem. 2015;290(40):24152-65.

79. Durk MR, et al. $1 \alpha, 25$-Dihydroxyvitamin D3 reduces cerebral amyloid- $\beta$ accumulation and improves cognition in mouse models of Alzheimer's disease. J Neurosci. 2014;34(21):7091-7101.

80. Combs B, Hamel C, Kanaan NM. Pathological conformations involving the amino terminus of tau occur early in Alzheimer's disease and are differentially detected by monoclonal antibodies. Neurobiol Dis. 2016;94:18-31.

81. Cox K, Combs B, Abdelmesih B, Morfini G, Brady ST, Kanaan NM. Analysis of isoform-specific tau aggregates suggests a common toxic mechanism involving similar pathological conformations and axonal transport inhibition. Neurobiol Aging. 2016;47:113-126.

82. Kanaan NM, Cox K, Alvarez VE, Stein TD, Poncil S, McKee AC. Characterization of early pathological tau conformations and phosphorylation in chronic traumatic encephalopathy. J Neuropathol Exp Neurol. 2016;75(1):19-34.

83. Combs B, Tiernan CT, Hamel C, Kanaan NM. Production of recombinant tau oligomers in vitro. Methods Cell Biol. 2017;141:45-64.

84. Ghoshal N, et al. Tau conformational changes correspond to impairments of episodic memory in mild cognitive impairment and Alzheimer's disease. Exp Neurol. 2002;177(2):475-493.

85. Patterson KR, et al. Characterization of prefibrillar Tau oligomers in vitro and in Alzheimer disease. J Biol Chem. 2011;286(26):23063-23076.

86. Ward SM, Himmelstein DS, Lancia JK, Fu Y, Patterson KR, Binder LI. TOC1: characterization of a selective oligomeric tau antibody. JAlzheimers Dis. 2013;37(3):593-602.

87. Berry RW, et al. Tau epitope display in progressive supranuclear palsy and corticobasal degeneration. J Neurocytol. 2004;33(3):287-295. 